

\title{
Reflexivity and rigidity for complexes, I Commutative rings
}

\author{
Luchezar L. Avramov, Srikanth B. lyengar and Joseph Lipman
}

To our friend and colleague, Hans-Bjфrn Foxby.

\begin{abstract}
A notion of rigidity with respect to an arbitrary semidualizing complex $C$ over a commutative noetherian ring $R$ is introduced and studied. One of the main results characterizes $C$-rigid complexes. Specialized to the case when $C$ is the relative dualizing complex of a homomorphism of rings of finite Gorenstein dimension, it leads to broad generalizations of theorems of Yekutieli and Zhang concerning rigid dualizing complexes, in the sense of Van den Bergh. Along the way, new results about derived reflexivity with respect to $C$ are established. Noteworthy is the statement that derived $C$-reflexivity is a local property; it implies that a finite $R$-module $M$ has finite $G$-dimension over $R$ if $M_{\mathfrak{m}}$ has finite $G$-dimension over $R_{\mathfrak{m}}$ for each maximal ideal $\mathfrak{m}$ of $R$.
\end{abstract}

Introduction $\quad 48$

1. Depth 50

2. Derived reflexivity 53

3. Semidualizing complexes $\quad 55$

4. Perfect complexes 57

5. Invertible complexes 62

6. Duality 67

7. Rigidity 70

8. Relative dualizing complexes 73

Appendix: Homological invariants $\quad 80$

Acknowledgments $\quad 84$

References $\quad 84$

MSC2000: primary 13D05, 13D25; secondary 13C15, $13 \mathrm{D} 03$.

Keywords: semidualizing complexes, perfect complexes, invertible complexes, rigid complexes, relative dualizing complexes, derived reflexivity, finite Gorenstein dimension.

Our research was partly supported by NSF grant DMS 0803082 (Avramov), NSF grant DMS 0602498 (Iyengar), and NSA grant H98230-06-1-0010 (Lipman). 


\section{Introduction}

Rigidification means, roughly, endowing a type of object with extra structure so as to eliminate nonidentity automorphisms. For example, a rigidification for dualizing sheaves on varieties over perfect fields plays an important role in [Lipman 1984]. We will be concerned with rigidifying complexes arising from Grothendieck duality theory, both in commutative algebra and in algebraic geometry. This paper is devoted to the algebraic situation; the geometric counterpart is treated in [Avramov et al. 2010b].

Let $R$ be a noetherian ring and $\mathrm{D}(R)$ its derived category. We write $\mathrm{D}_{\mathrm{b}}^{\mathrm{f}}(R)$ for the full subcategory of homologically finite complexes, that is to say, complexes $M$ for which the $R$-module $\mathrm{H}(M)$ is finitely generated. Given complexes $M$ and $C$ in $\mathrm{D}_{\mathrm{b}}^{\mathrm{f}}(R)$ one says that $M$ is derived $C$-reflexive if the canonical map

$$
\delta_{M}^{C}: M \longrightarrow \mathrm{RHom}_{R}\left(\mathrm{RHom}_{R}(M, C), C\right)
$$

is an isomorphism and $\operatorname{RHom}_{R}(M, C)$ is homologically finite. When the ring $R$ has finite Krull dimension, the complex $C$ is said to be dualizing for $R$ if $\delta_{M}^{C}$ is an isomorphism for all homologically finite complexes $M$. In [Hartshorne 1966, p. 258, 2.1] it is proved that when $C$ is isomorphic to some bounded complex of injective modules, $C$ is dualizing if and only if it is semidualizing, meaning that the canonical map

$$
\chi^{C}: R \longrightarrow \mathrm{RHom}_{R}(C, C)
$$

is an isomorphism.

Even when Spec $R$ is connected, dualizing complexes for $R$ differ by shifts and the action of the Picard group of the ring [Hartshorne 1966, p. 266, 3.1]. Such a lack of uniqueness has been a source of difficulties. Building on work of Van den Bergh [1997] and extensively using differential graded algebras, Yekutieli and Zhang [2008; 2009] have developed for algebras of finite type over a regular ring $K$ of finite Krull dimension a theory of rigid relative to $K$ dualizing complexes. The additional structure that they carry makes them unique up to unique rigid isomorphism.

Our approach to rigidity applies to any noetherian ring $R$ and takes place entirely within its derived category: We say that $M$ is $C$-rigid if there is an isomorphism

$$
\mu: M \stackrel{\simeq}{\longrightarrow} \operatorname{RHom}_{R}\left(\operatorname{RHom}_{R}(M, C), M\right),
$$

called a $C$-rigidifying isomorphism for $M$. In the context described in the previous paragraph we prove, using the main result of [Avramov et al. 2010a], that rigidity in the sense of Van den Bergh, Yekutieli, and Zhang coincides with $C$-rigidity for a specific complex $C$. 
The precise significance of $C$-rigidity is explained by the following result. It is abstracted from Theorem 7.3, which requires no connectedness hypothesis.

Theorem 1. If $C$ is a semidualizing complex, then $\operatorname{RHom}_{R}\left(\chi^{C}, C\right)^{-1}$ is a $C$ rigidifying isomorphism.

When Spec $R$ is connected and $M$ is nonzero and $C$-rigid, with $C$-rigidifying isomorphism $\mu$, there exists a unique isomorphism $\alpha: C \stackrel{\sim}{\longrightarrow}$ M making the following diagram commute:

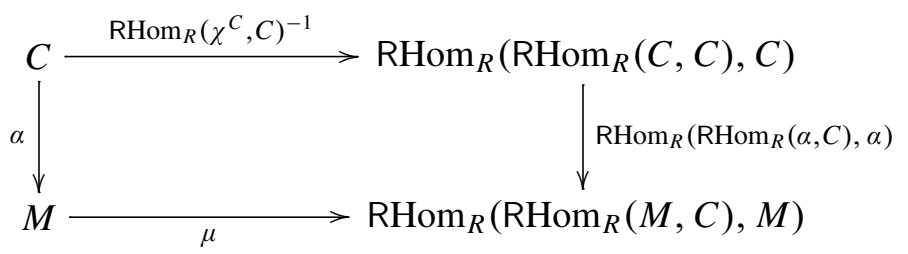

Semidualizing complexes, identified by Foxby [1972] and Golod [1984] in the case of modules, have received considerable attention in [Avramov and Foxby 1997] and in the work of Christensen, Frankild, Sather-Wagstaff, and Taylor [Christensen 2001; Frankild and Sather-Wagstaff 2007; Frankild et al. 2009]. However, to achieve our goals we need to go further back and rethink basic propositions concerning derived reflexivity. This is the content of Sections 1 through 6, from which we highlight some results.

Theorem 2. When $C$ is semidualizing, $M$ is derived $C$-reflexive if (and only if) there exists some isomorphism $M \simeq \mathrm{RHom}_{R}\left(\operatorname{RHom}_{R}(M, C), C\right)$ in $\mathrm{D}(R)$, if (and only if) $M_{\mathfrak{m}}$ is derived $C_{\mathfrak{m}}$-reflexive for each maximal ideal $\mathfrak{m}$ of $R$.

This is part of Theorem 3.3. One reason for its significance is that it delivers derived $C$-reflexivity bypassing a delicate step, the verification that $\operatorname{RHom}_{R}(M, C)$ is homologically finite. Another is that it establishes that derived $C$-reflexivity is a local property. This implies, in particular, that a finite $R$-module $M$ has finite G-dimension (Gorenstein dimension) in the sense of Auslander and Bridger [1969] if it has that property at each maximal ideal of $R$; see Corollary 6.3.4.

In Theorem 5.6 we characterize pairs of mutually reflexive complexes:

Theorem 3. The complexes $C$ and $M$ are semidualizing and satisfy $C \simeq L \otimes_{R} M$ for some invertible graded $R$-module $L$ if and only if $M$ is derived $C$-reflexive, $C$ is derived $M$-reflexive, and $\mathrm{H}(M)_{\mathfrak{p}} \neq 0$ holds for every $\mathfrak{p} \in \operatorname{Spec} R$.

In the last section we apply our results to the relative dualizing complex $D^{\sigma}$ attached to an algebra $\sigma: K \rightarrow S$ essentially of finite type over a noetherian ring $K$; see [Avramov et al. 2010a, 1.1 and 6.2]. We show that $D^{\sigma}$ is semidualizing if and only if $\sigma$ has finite G-dimension in the sense of [Avramov and Foxby 1997]. One case when the G-dimension of $\sigma$ is finite is if $S$ has finite flat dimension as a 
$K$-module. In this context, it follows from a result of [Avramov et al. 2010a] that $D^{\sigma}$-rigidity is equivalent to rigidity relative to $K$, in the sense of [Yekutieli and Zhang 2009]. We prove:

Theorem 4. If $K$ is Gorenstein, the flat dimension of the $K$-module $S$ is finite, and $\operatorname{dim} S$ is finite, then $D^{\sigma}$ is dualizing for $S$ and is rigid relative to $K$.

When moreover Spec $S$ is connected, $D^{\sigma}$ is the unique, up to unique rigid isomorphism, nonzero complex in $\mathrm{D}_{\mathrm{b}}^{\mathrm{f}}(S)$ that is rigid relative to $K$.

This result, which is contained in Theorem 8.5.6, applies in particular when $K$ is regular, and is a broad generalization of one of the main results in [Yekutieli and Zhang 2009].

Our terminology and notation are mostly in line with literature in commutative algebra. In particular, we put "homological" gradings on complexes, so at first sight some formulas may look unfamiliar to experts used to cohomological conventions. More details may be found in the Appendix, where we also prove results on Poincaré series and Bass series of complexes invoked repeatedly in the body of the text.

Several objects studied in this paper were introduced by Hans-Bjørn Foxby, and various techniques used below were initially developed by him. We have learned a lot about the subject from his articles, his lectures, and through collaborations with him. This work is dedicated to him in appreciation and friendship.

\section{Depth}

Throughout the paper $R$ denotes a commutative noetherian ring. An $R$-module is said to be "finite" if it can be generated, as an $R$-module, by finitely many elements.

The depth of a complex $M$ over a local ring $R$ with residue field $k$ is the number

$$
\operatorname{depth}_{R} M=\inf \left\{n \in \mathrm{Z} \mid \operatorname{Ext}_{R}^{n}(k, M) \neq 0\right\} .
$$

We focus on a global invariant that appears in work of Chouinard and Foxby:

$$
\operatorname{Rfd}_{R} M=\sup \left\{\operatorname{depth} R_{\mathfrak{p}}-\operatorname{depth}_{R_{\mathfrak{p}}} M_{\mathfrak{p}} \mid \mathfrak{p} \in \operatorname{Spec} R\right\} .
$$

See 1.6 for a different description of this number. Our goal is to prove:

Theorem 1.1. Every complex $M$ in $\mathrm{D}_{\mathrm{b}}^{\mathrm{f}}(R)$ satisfies $\operatorname{Rfd}_{R} M<\infty$.

The desired inequality is obvious for rings of finite Krull dimension. To handle the general case, we adapt the proof of a result of Gabber; see Proposition 1.5.

A couple of simple facts are needed to keep the argument going:

1.2. If $0 \rightarrow L \rightarrow M \rightarrow N \rightarrow 0$ is an exact sequence of complexes then one has

$$
\operatorname{Rfd}_{R} M \leq \max \left\{\operatorname{Rfd}_{R} L, \operatorname{Rfd}_{R} N\right\} .
$$


Indeed, for every $\mathfrak{p} \in \operatorname{Spec} R$ and each $n \in \mathbb{Z}$ one has an induced exact sequence

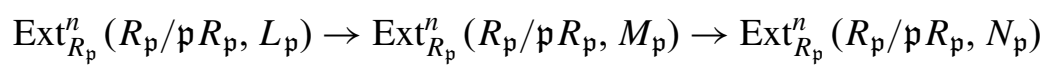

that yields depth ${ }_{R_{\mathfrak{p}}} M_{\mathfrak{p}} \geq \min \left\{\operatorname{depth}_{R_{\mathfrak{p}}} L_{\mathfrak{p}}, \operatorname{depth}_{R_{\mathfrak{p}}} N_{\mathfrak{p}}\right\}$.

The statement below is an Auslander-Buchsbaum equality for complexes:

1.3. Each bounded complex $F$ of finite free modules over a local ring $R$ has

$$
\operatorname{depth}_{R} F=\operatorname{depth} R-\sup \mathrm{H}\left(k \otimes_{R} F\right) ;
$$

see [Foxby 1979, 3.13]. This formula is an immediate consequence of the isomorphisms

$$
\mathrm{RHom}_{R}(k, F) \simeq \operatorname{RHom}_{R}(k, R) \otimes_{R}^{\mathrm{L}} F \simeq \operatorname{RHom}_{R}(k, R) \otimes_{k}^{\mathrm{L}}\left(k \otimes_{R} F\right)
$$

in $\mathrm{D}(R)$, where the first one holds because $F$ is finite free.

Proof of Theorem 1.1. It's enough to prove that $\operatorname{Rfd}_{R} M<\infty$ holds for cyclic modules. Indeed, replacing $M$ with a quasiisomorphic complex we may assume $\operatorname{amp} M=\operatorname{amp} \mathrm{H}(M)$. If one has amp $M=0$, then $M$ is a shift of a finite $R$-module, so an induction on the number of its generators, using 1.2 , shows that $\operatorname{Rfd}_{R} M$ is finite. Assume the statement holds for all complexes of a given amplitude. Since $L=\Sigma^{i} M_{i}$ with $i=\inf M$ is a subcomplex of $M$, and one has $\operatorname{amp}(M / L)<\operatorname{amp} M$, using 1.2 and induction we obtain $\operatorname{Rfd}_{R} M \leq \max \left\{\operatorname{Rfd}_{R} L, \operatorname{Rfd}_{R}(M / L)\right\}<\infty$.

By way of contradiction, assume $\operatorname{Rfd}_{R}(R / J)=\infty$ holds for some ideal $J$ of $R$. Since $R$ is noetherian, we may choose $J$ so that $\operatorname{Rfd}_{R}(R / I)$ is finite for each ideal $I$ with $I \supsetneq J$. The ideal $J$ is prime: otherwise one would have an exact sequence

$$
0 \rightarrow R / J^{\prime} \rightarrow R / J \rightarrow R / I \rightarrow 0,
$$

where $J^{\prime}$ is a prime ideal associated to $R / J$ with $J^{\prime} \supsetneq J$; this implies $I \supsetneq J$, so in view of 1.2 the exact sequence yields $\operatorname{Rfd}_{R}(R / J)<\infty$, which is absurd.

Set $S=R / J$, fix a finite generating set of $J$, let $g$ denote its cardinality, and $E$ be the Koszul complex on it. As $S$ is a domain and $\bigoplus_{i} \mathrm{H}_{i}(E)$ is a finite $S$-module, we may choose $f \in R \backslash J$ so that each $S_{f}$-module $\mathrm{H}_{i}(E)_{f}$ is free. Now $(J, f) \supsetneq J$ implies that $j=\operatorname{Rfd}_{R}(R /(J, f))$ is finite. To get the desired contradiction we prove

$$
\text { depth } R_{\mathfrak{p}}-\operatorname{depth}_{R_{\mathfrak{p}}} S_{\mathfrak{p}} \leq \max \{j-1, g\} \quad \text { for each } \mathfrak{p} \in \operatorname{Spec} R \text {. }
$$

In the case $\mathfrak{p} \nsupseteq J$ one has depth $R_{R_{\mathfrak{p}}} S_{\mathfrak{p}}=\infty$, so the inequality obviously holds. When $\mathfrak{p} \supseteq(J, f)$ the exact sequence

$$
0 \rightarrow S \stackrel{f}{\rightarrow} S \rightarrow R /(J, f) \rightarrow 0
$$


yields depth $_{R_{\mathfrak{p}}} S_{\mathfrak{p}}=\operatorname{depth}_{R_{\mathfrak{p}}}(R /(J, f))_{\mathfrak{p}}+1$, and hence one has

$$
\operatorname{depth} R_{\mathfrak{p}}-\operatorname{depth}_{R_{\mathfrak{p}}} S_{\mathfrak{p}} \leq j-1 \text {. }
$$

It remains to treat the case $f \notin \mathfrak{p} \supseteq J$. Set $k=R_{\mathfrak{p}} / \mathfrak{p} R_{\mathfrak{p}}, d=\operatorname{depth}_{R_{\mathfrak{p}}} S_{\mathfrak{p}}$, and $s=\sup \mathrm{H}\left(E_{\mathfrak{p}}\right)$. In the second quadrant spectral sequence

$$
\begin{aligned}
{ }^{2} \mathrm{E}_{p, q}=\operatorname{Ext}_{R_{\mathfrak{p}}}^{-p}\left(k, \mathrm{H}_{q}\left(E_{\mathfrak{p}}\right)\right) & \Longrightarrow \operatorname{Ext}_{R_{\mathfrak{p}}}^{-p-q}\left(k, E_{\mathfrak{p}}\right), \\
{ }^{r} \mathrm{~d}^{p, q}:{ }^{r} \mathrm{E}_{p, q} & \longrightarrow{ }^{r} \mathrm{E}_{p-r, q+r-1},
\end{aligned}
$$

one has ${ }^{2} \mathrm{E}_{p, q}=0$ for $q>s$, and also for $p>-d$ because each $\mathrm{H}_{q}\left(E_{\mathfrak{p}}\right)$ is a finite direct sum of copies of $S_{\mathfrak{p}}$. Therefore, the sequence converges strongly and yields

$$
\operatorname{Ext}_{R_{\mathfrak{p}}}^{i}\left(k, E_{\mathfrak{p}}\right) \cong \begin{cases}0 & \text { for } i<d-s, \\ \operatorname{Ext}_{R_{\mathfrak{p}}}^{d}\left(k, \mathrm{H}_{s}\left(E_{\mathfrak{p}}\right)\right) \neq 0 & \text { for } i=d-s .\end{cases}
$$

The formula above implies depth $R_{R_{\mathfrak{p}}} E_{\mathfrak{p}}=d-s$. This gives the first equality below, with the second equality coming from 1.3 .

$$
\begin{aligned}
\operatorname{depth} R_{\mathfrak{p}}-\operatorname{depth}_{R_{\mathfrak{p}}} S_{\mathfrak{p}} & =\operatorname{depth} R_{\mathfrak{p}}-\operatorname{depth}_{R_{\mathfrak{p}}} E_{\mathfrak{p}}-s \\
& =\operatorname{supH}\left(k \otimes_{R_{\mathfrak{p}}} E_{\mathfrak{p}}\right)-s \\
& \leq g-s \leq g .
\end{aligned}
$$

A complex in $D_{-}(R)$ is said to have finite injective dimension if it is isomorphic in $\mathrm{D}(R)$ to a bounded complex of injective $R$-modules. The next result, due to Ischebeck $[1969,2.6]$ when $M$ and $N$ are modules, can be deduced from [Christensen et al. 2002, 4.13].

Lemma 1.4. Let $R$ be a local ring and $N$ in $\mathrm{D}_{\mathrm{b}}^{\mathrm{f}}(R)$ a complex of finite injective dimension. For each $M$ in $\mathrm{D}_{\mathrm{b}}^{\mathrm{f}}(R)$ there is an equality

$$
\sup \left\{n \in \mathrm{Z} \mid \operatorname{Ext}_{R}^{n}(M, N) \neq 0\right\}=\operatorname{depth} R-\operatorname{depth} M-\inf \mathrm{H}(N) .
$$

Proof. Let $k$ be the residue field $k$ of $R$. The first isomorphism below holds because $N$ has finite injective dimension and $M$ is in $\mathrm{D}_{\mathrm{b}}^{\mathrm{f}}(R)$ (see [Avramov and Foxby 1991, 4.4.I]):

$$
\begin{aligned}
\mathrm{H}\left(k \otimes_{R}^{\mathrm{L}} \operatorname{RHom}_{R}(M, N)\right) & \cong \mathrm{H}\left(\operatorname{RHom}_{R}\left(\operatorname{RHom}_{R}(k, M), N\right)\right) \\
& \cong \mathrm{H}\left(\operatorname{RHom}_{k}\left(\operatorname{RHom}_{R}(k, M), \operatorname{RHom}_{R}(k, N)\right)\right) \\
& \cong \operatorname{Hom}_{k}\left(\operatorname{H}\left(\operatorname{RHom}_{R}(k, M)\right), \operatorname{H}\left(\operatorname{RHom}_{R}(k, N)\right)\right) .
\end{aligned}
$$

The other isomorphisms are standard. One deduces the second equality below:

$$
\begin{aligned}
\inf \mathrm{H}\left(\operatorname{RHom}_{R}(M, N)\right) & =\inf \mathrm{H}\left(k \otimes_{R}^{\mathrm{L}} \operatorname{RHom}_{R}(M, N)\right) \\
& =\inf \mathrm{H}\left(\operatorname{RHom}_{R}(k, N)\right)+\operatorname{depth}_{R} M .
\end{aligned}
$$


The first one comes from Lemma A.4.3. In particular, for $M=R$ this yields

$$
\inf \mathrm{H}\left(\mathrm{RHom}_{R}(k, N)\right)=\inf \mathrm{H}(N)-\operatorname{depth} R .
$$

Combining the preceding equalities, one obtains the desired assertion.

The next result is due to Gabber [Conrad 2000, 3.1.5]; Goto [1982] had proved it for $N=R$.

Proposition 1.5. For each $N$ in $\mathrm{D}_{\mathrm{b}}^{\mathrm{f}}(R)$ the following conditions are equivalent.

(i) For each $\mathfrak{p} \in \operatorname{Spec} R$ the complex $N_{\mathfrak{p}}$ has finite injective dimension over $R_{\mathfrak{p}}$.

(ii) For each $M$ in $\mathrm{D}_{\mathrm{b}}^{\mathrm{f}}(R)$ one has $\operatorname{Ext}_{R}^{n}(M, N)=0$ for $n \gg 0$.

(ii') For each $\mathfrak{m} \in \operatorname{Max} R$ one has $\operatorname{Ext}_{R}^{n}(R / \mathfrak{m}, N)=0$ for $n \gg 0$.

Proof. (i) $\Longrightarrow$ (ii). For each prime $\mathfrak{p}$, Lemma 1.4 yields the second equality below:

$$
\begin{aligned}
-\operatorname{infH}\left(\operatorname{RHom}_{R}(M, N)_{\mathfrak{p}}\right) & =-\inf \mathrm{H}\left(\operatorname{RHom}_{R_{\mathfrak{p}}}\left(M_{\mathfrak{p}}, N_{\mathfrak{p}}\right)\right) \\
& =\operatorname{depth} R_{\mathfrak{p}}-\operatorname{depth}_{R_{\mathfrak{p}}} M_{\mathfrak{p}}-\inf \mathrm{H}\left(N_{\mathfrak{p}}\right) \\
& \leq \operatorname{Rfd}_{R} M-\inf \mathrm{H}(N) .
\end{aligned}
$$

Theorem 1.1 thus implies the desired result.

$\left(\right.$ ii $\left.^{\prime}\right) \Longrightarrow$ (i). Since $N$ is in $\mathrm{D}_{\mathrm{b}}^{\mathrm{f}}(R)$ for each integer $n$, one has an isomorphism

$$
\operatorname{Ext}_{R_{\mathfrak{m}}}^{n}\left(R_{\mathfrak{m}} / \mathfrak{m} R_{\mathfrak{m}}, N_{\mathfrak{m}}\right) \cong \operatorname{Ext}_{R}^{n}(R / \mathfrak{m}, N)_{\mathfrak{m}} .
$$

Thus, the hypothesis and A.5.1 imply $N_{\mathfrak{m}}$ has finite injective dimension over $R_{\mathfrak{m}}$. By localization, $N_{\mathfrak{p}}$ has finite injective dimension over $R_{\mathfrak{p}}$ for each prime $\mathfrak{p} \subseteq \mathfrak{m}$.

Notes 1.6. In [Christensen et al. 2002, 2.1] the number $\operatorname{Rfd}_{R} M$ is defined by the formula

$$
\operatorname{Rfd}_{R} M=\sup \left\{n \in \mathbb{Z} \mid \operatorname{Tor}_{n}^{R}(T, M) \neq 0\right\},
$$

where $T$ ranges over the $R$-modules of finite flat dimension, and is called the large restricted flat dimension of $M$ (hence the notation). With this definition, formula (1.0.1) is due to Foxby (see [Christensen 2000, Notes, p. 131]) and is proved in [Christensen 2000, 5.3.6; Christensen et al. 2002, 2.4(b)]. For $M$ of finite flat dimension one has $\operatorname{Rfd}_{M}=\mathrm{fd}_{R} M$ [Christensen 2000, 5.4.2(b); Christensen et al. 2002, 2.5] and then (1.0.1) goes back to [Chouinard 1976, 1.2].

\section{Derived reflexivity}

For every pair $C, M$ in $\mathrm{D}(R)$ there is a canonical biduality morphism

$$
\delta_{M}^{C}: M \rightarrow \mathrm{RHom}_{R}\left(\mathrm{RHom}_{R}(M, C), C\right),
$$


induced by the morphism of complexes $m \mapsto\left(\alpha \mapsto(-1)^{|m||\alpha|} \alpha(m)\right)$. We say that $M$ is derived $C$-reflexive if both $M$ and $\operatorname{RHom}_{R}(M, C)$ are in $\mathrm{D}_{\mathrm{b}}^{\mathrm{f}}(R)$, and $\delta_{M}^{C}$ is an isomorphism. Some authors write " $C$-reflexive" instead of "derived $C$-reflexive".

Recall that the support of a complex $M$ in $\mathrm{D}_{\mathrm{b}}^{\mathrm{f}}(R)$ is the set

$$
\operatorname{Supp}_{R} M=\left\{\mathfrak{p} \in \operatorname{Spec} R \mid \mathrm{H}(M)_{\mathfrak{p}} \neq 0\right\} .
$$

Theorem 2.1. Let $R$ be a noetherian ring and $C$ a complex in $\mathrm{D}_{\mathrm{b}}^{\mathrm{f}}(R)$.

For each complex $M$ in $\mathrm{D}_{\mathrm{b}}^{\mathrm{f}}(R)$, the following conditions are equivalent.

(i) $M$ is derived $C$-reflexive.

(ii) $\operatorname{RHom}_{R}(M, C)$ is derived $C$-reflexive and $\operatorname{Supp}_{R} M \subseteq \operatorname{Supp}_{R} C$ holds.

(iii) $\operatorname{RHom}_{R}(M, C)$ is in $\mathrm{D}_{+}(R)$, and for every $\mathfrak{m} \in \operatorname{Max} R$ one has

$$
M_{\mathfrak{m}} \simeq \operatorname{RHom}_{R_{\mathfrak{m}}}\left(\operatorname{RHom}_{R_{\mathfrak{m}}}\left(M_{\mathfrak{m}}, C_{\mathfrak{m}}\right), C_{\mathfrak{m}}\right) \quad \text { in } \mathrm{D}\left(R_{\mathfrak{m}}\right) .
$$

(iv) $U^{-1} M$ is derived $U^{-1} C$-reflexive for each multiplicatively closed set $U \subseteq R$.

The proof is based on a useful criterion for derived $C$-reflexivity.

2.2. Let $C$ and $M$ be complexes of $R$-modules, and set $\mathrm{h}=\mathrm{RHom}_{R}(-, C)$.

The composition $\mathrm{h}\left(\delta_{M}^{C}\right) \circ \delta_{\mathrm{h}(M)}^{C}$ is the identity map of $\mathrm{h}(M)$, so the map

$$
\mathrm{H}\left(\delta_{\mathrm{h}(M)}^{C}\right): \mathrm{H}(\mathrm{h}(M)) \rightarrow \mathrm{H}\left(\mathrm{h}^{3}(M)\right)
$$

is a split monomorphism. Thus, if $\mathrm{h}(M)$ is in $\mathrm{D}^{\mathrm{f}}(R)$ and there exists some isomorphism $\mathrm{H}(\mathrm{h}(M)) \cong \mathrm{H}\left(\mathrm{h}^{3}(M)\right)$, then $\delta_{\mathrm{h}(M)}^{C}$ and $\mathrm{h}\left(\delta_{M}^{C}\right)$ are isomorphisms in $\mathrm{D}(R)$.

The following proposition is an unpublished result of Foxby.

Proposition 2.3. If for $C$ and $M$ in $\mathrm{D}_{\mathrm{b}}^{\mathrm{f}}(R)$ there exists an isomorphism

$$
\mu: M \simeq \operatorname{RHom}_{R}\left(\operatorname{RHom}_{R}(M, C), C\right) \quad \text { in } \mathrm{D}(R),
$$

then the biduality morphism $\delta_{M}^{C}$ is an isomorphism as well.

Proof. Set $\mathrm{h}=\mathrm{RHom}_{R}(-, C)$. Note that $\mathrm{h}(M)$ is in $\mathrm{D}_{-}^{\mathrm{f}}(R)$ because $C$ and $M$ are in $\mathrm{D}_{\mathrm{b}}^{\mathrm{f}}(R)$. The morphism $\mu$ induces an isomorphism $\mathrm{H}\left(\mathrm{h}^{3}(M)\right) \cong \mathrm{H}(\mathrm{h}(M))$. Each $R$-module $\mathrm{H}_{n}(\mathrm{~h}(M))=\operatorname{Ext}_{R}^{-n}(M, C)$ is finite, so we conclude from 2.2 that $\delta_{\mathrm{h}(M)}^{C}$ is an isomorphism in $\mathrm{D}(R)$, and hence $\delta_{\mathrm{h}^{2}(M)}^{C}$ is one as well. The square

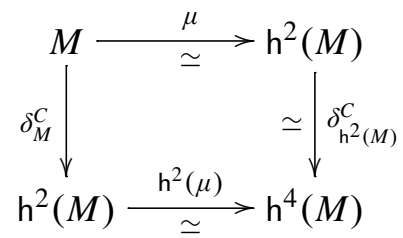

in $\mathrm{D}(R)$ commutes and implies that $\delta_{M}^{C}$ is an isomorphism, as desired. 
Proof of Theorem 2.1. (i) $\Longrightarrow$ (ii). This follows from 2.2 and A.6.

(ii) $\Longrightarrow$ (i). Set $\mathrm{h}=\mathrm{RHom}_{R}(-, C)$ and form the exact triangle in $\mathrm{D}(R)$ :

$$
M \stackrel{\delta_{M}^{C}}{\longrightarrow} \mathrm{h}^{2}(M) \longrightarrow N \longrightarrow
$$

As $\mathrm{h}(M)$ is $C$-reflexive, one has $\mathrm{h}^{2}(M) \in \mathrm{D}_{\mathrm{b}}^{\mathrm{f}}(R)$, so the exact triangle above implies that $N$ is in $\mathrm{D}_{\mathrm{b}}^{\mathrm{f}}(R)$. Since $\operatorname{Supp}_{R} M \subseteq \operatorname{Supp}_{R} C$ holds, using A.6 one obtains

$$
\begin{aligned}
\operatorname{Supp}_{R} N & \subseteq \operatorname{Supp}_{R} M \cup \operatorname{Supp}_{R} h^{2}(M) \\
& =\operatorname{Supp}_{R} M \cup\left(\operatorname{Supp}_{R} M \cap \operatorname{Supp}_{R} C\right) \subseteq \operatorname{Supp}_{R} C .
\end{aligned}
$$

On the other hand, the exact triangle above induces an exact triangle

$$
\mathrm{h}(N) \longrightarrow \mathrm{h}^{3}(M) \stackrel{\mathrm{h}\left(\delta_{M}^{C}\right)}{\longrightarrow} \mathrm{h}(M) \longrightarrow
$$

Since $\mathrm{h}(M)$ is $C$-reflexive $\delta_{\mathrm{h}(M)}^{C}$ is an isomorphism, so 2.2 shows that $\mathrm{h}\left(\delta_{M}^{C}\right)$ is an isomorphism as well. The second exact triangle now gives $\mathrm{H}(\mathrm{h}(N))=0$. The already established inclusion $\operatorname{Supp}_{R} N \subseteq \operatorname{Supp}_{R} C$ and A.6 yield

$$
\operatorname{Supp}_{R} N=\operatorname{Supp}_{R} N \cap \operatorname{Supp}_{R} C=\operatorname{Supp}_{R} \operatorname{RHom}_{R}(N, C)=\varnothing .
$$

This implies $N=0$ in $\mathrm{D}(R)$, and hence $\delta_{M}^{C}$ is an isomorphism.

(i) $\Longrightarrow$ (iv). This is a consequence of the hypothesis $\operatorname{RHom}_{R}(M, C) \in \mathrm{D}_{+}^{f}(R)$.

(iv) $\Longrightarrow$ (iii). With $U=\{1\}$ the hypotheses in (iv) implies $\operatorname{RHom}_{R}(M, C)$ is in $\mathrm{D}_{+}^{\mathrm{f}}(R)$, while the isomorphism in (iii) is the special case $U=R \backslash \mathfrak{m}$.

(iii) $\Longrightarrow$ (i). For each $\mathfrak{m} \in \operatorname{Max} R$, Proposition 2.3 yields that $\delta_{M_{\mathfrak{m}}}^{C_{\mathfrak{m}}}$ is an isomorphism, in $\mathrm{D}\left(R_{\mathfrak{m}}\right)$. One has a canonical isomorphism

$$
\lambda_{\mathfrak{m}}: \operatorname{RHom}_{R}\left(\operatorname{RHom}_{R}(M, C), C\right)_{\mathfrak{m}} \stackrel{\simeq}{\rightarrow} \operatorname{RHom}_{R_{\mathfrak{m}}}\left(\operatorname{RHom}_{R_{\mathfrak{m}}}\left(M_{\mathfrak{m}}, C_{\mathfrak{m}}\right), C_{\mathfrak{m}}\right),
$$

because $\operatorname{RHom}_{R}(M, C)$ is in $\mathrm{D}_{+}^{\mathrm{f}}(R)$ and $M$ is in $\mathrm{D}_{\mathrm{b}}^{\mathrm{f}}(R)$. Now using the equality $\delta_{M_{\mathfrak{m}}}^{C_{\mathfrak{m}}}=\lambda_{\mathfrak{m}}\left(\delta_{M}^{C}\right)_{\mathfrak{m}}$ one sees that $\left(\delta_{M}^{C}\right)_{\mathfrak{m}}$ is an isomorphism, and hence so is $\delta_{M}^{C}$.

\section{Semidualizing complexes}

For each complex $C$ there is a canonical homothety morphism

$$
\chi^{C}: R \rightarrow \operatorname{RHom}_{R}(C, C) \quad \text { in } \mathrm{D}(R)
$$

induced by $r \mapsto(c \mapsto r c)$. As in [Christensen 2001, 2.1], we say that $C$ is semidualizing if it is in $\mathrm{D}_{\mathrm{b}}^{\mathrm{f}}(R)$ and $\chi^{C}$ an isomorphism. We bundle convenient recognition criteria in:

Proposition 3.1. For a complex $C$ in $\mathrm{D}_{\mathrm{b}}^{\mathrm{f}}(R)$ the following are equivalent:

(i) $C$ is semidualizing. 
(i') $R$ is derived $C$-reflexive.

(ii) $C$ is derived $C$-reflexive and $\operatorname{Supp}_{R} C=\operatorname{Spec} R$.

(iii) For each $\mathfrak{m} \in \operatorname{Max} R$ there is an isomorphism

$$
R_{\mathfrak{m}} \simeq \operatorname{RHom}_{R_{\mathfrak{m}}}\left(C_{\mathfrak{m}}, C_{\mathfrak{m}}\right) \quad \text { in } \mathrm{D}\left(R_{\mathfrak{m}}\right) .
$$

(iv) $U^{-1} C$ is semidualizing for $U^{-1} R$ for each multiplicatively closed set $U \subseteq R$. Proof. To see that (i) and (i') are equivalent, decompose $\chi^{C}$ as

$$
R \stackrel{\delta_{R}^{C}}{\longrightarrow} \operatorname{RHom}_{R}\left(\operatorname{RHom}_{R}(R, C), C\right) \stackrel{\simeq}{\rightarrow} \operatorname{RHom}_{R}(C, C),
$$

with isomorphism induced by the canonical isomorphism $C \stackrel{\simeq}{\rightarrow} \operatorname{RHom}_{R}(R, C)$. Conditions (i') through (iv) are equivalent by Theorem 2.1 applied with $M=R$.

Next we establish a remarkable property of semidualizing complexes. It uses the invariant $\operatorname{Rfd}_{R}(-)$ discussed in Section 1.

Theorem 3.2. If $C$ is a semidualizing complex for $R$ and $L$ is a complex in $\mathrm{D}_{-}^{\mathrm{f}}(R)$ with $\operatorname{RHom}_{R}(L, C) \in \mathrm{D}_{\mathrm{b}}^{\mathrm{f}}(R)$, then $L$ is in $\mathrm{D}_{\mathrm{b}}^{\mathrm{f}}(R)$; more precisely, one has

$$
\inf \mathrm{H}(L) \geq \inf \mathrm{H}(C)-\operatorname{Rfd}_{R} \operatorname{RHom}_{R}(L, C)>-\infty .
$$

Proof. For each $\mathfrak{m} \in \operatorname{Max} R \cap \operatorname{Supp}_{R} L$ one has a chain of relations

$$
\begin{aligned}
\inf \mathrm{H}\left(L_{\mathfrak{m}}\right) & =-\operatorname{depth}_{R_{\mathfrak{m}}} C_{\mathfrak{m}}+\operatorname{depth}_{R_{\mathfrak{m}}} \operatorname{RHom}_{R}(L, C)_{\mathfrak{m}} \\
& =\inf \mathrm{H}\left(C_{\mathfrak{m}}\right)-\operatorname{depth} R_{\mathfrak{m}}+\operatorname{depth}_{R_{\mathfrak{m}}} \operatorname{RHom}_{R}(L, C)_{\mathfrak{m}} \\
& \geq \inf \mathrm{H}(C)-\operatorname{Rfd}_{R} \operatorname{RHom}_{R}(L, C) \\
& >-\infty
\end{aligned}
$$

with equalities given by Lemma A.5.3, applied first with $M=L$ and $N=C$, then with $M=C=N$; the first inequality is clear, and the second one holds by Theorem 1.1. Now use the equality $\inf \mathrm{H}(L)=\inf _{\mathfrak{m} \in \operatorname{Max} R}\left\{\inf \mathrm{H}\left(L_{\mathfrak{m}}\right)\right\}$.

The next theorem parallels Theorem 2.1. The impact of the hypothesis that $C$ is semidualizing can be seen by comparing condition (iii) in these results: one need not assume $\operatorname{RHom}_{R}(M, C)$ is bounded. In particular, reflexivity with respect to a semidualizing complex can now be defined by means of property ( $\left.\mathrm{i}^{\prime}\right)$ alone. Antecedents of the theorem are discussed in 3.4.

Theorem 3.3. Let $C$ be a semidualizing complex for $R$.

For a complex $M$ in $\mathrm{D}_{\mathrm{b}}^{\mathrm{f}}(R)$ the following conditions are equivalent:

(i) $M$ is derived $C$-reflexive.

(i') There exists an isomorphism $M \simeq \mathrm{RHom}_{R}\left(\mathrm{RHom}_{R}(M, C), C\right)$. 
(ii) $\operatorname{RHom}_{R}(M, C)$ is derived $C$-reflexive.

(iii) For each $\mathfrak{m} \in \operatorname{Max} R$ there is an isomorphism

$$
M_{\mathfrak{m}} \simeq \operatorname{RHom}_{R_{\mathfrak{m}}}\left(\operatorname{RHom}_{R_{\mathfrak{m}}}\left(M_{\mathfrak{m}}, C_{\mathfrak{m}}\right), C_{\mathfrak{m}}\right) \quad \text { in } \mathrm{D}\left(R_{\mathfrak{m}}\right) .
$$

Furthermore, these conditions imply the following inequalities

$$
\operatorname{amp} \mathrm{H}\left(\operatorname{RHom}_{R}(M, C)\right) \leq \operatorname{ampH}(C)-\inf \mathrm{H}(M)+\operatorname{Rfd}_{R} M<\infty .
$$

Proof. (i) $\Longleftrightarrow$ (ii). Apply Theorem 2.1, noting that $\operatorname{Supp}_{R} C=\operatorname{Spec} R$, by A.6.

(i) $\Longrightarrow\left(i^{\prime}\right)$. This implication is a tautology.

$\left(\mathrm{i}^{\prime}\right) \Longrightarrow$ (iii). This holds because Theorem 3.2, applied with $L=\operatorname{RHom}_{R}(M, C)$, shows that $\operatorname{RHom}_{R}(M, C)$ is bounded, and so the given isomorphism localizes.

(iii) $\Longrightarrow$ (i). For each $\mathfrak{m} \in \operatorname{Max} R$ the complex $C_{\mathfrak{m}}$ is semidualizing for $R_{\mathfrak{m}}$ by Proposition 3.1. One then has a chain of (in)equalities

$$
\begin{aligned}
\inf \mathrm{H}\left(\operatorname{RHom}_{R}(M, C)\right) & =\inf _{\mathfrak{m} \in \operatorname{Max} R}\left\{\operatorname{infH}\left(\operatorname{RHom}_{R}(M, C)_{\mathfrak{m}}\right)\right\} \\
& =\inf _{\mathfrak{m} \in \operatorname{Max} R}\left\{\inf \mathrm{H}\left(\operatorname{RHom}_{R_{\mathfrak{m}}}\left(M_{\mathfrak{m}}, C_{\mathfrak{m}}\right)\right)\right\} \\
& \geq \inf _{\mathfrak{m} \in \operatorname{Max} R}\left\{\inf \mathrm{H}\left(C_{\mathfrak{m}}\right)-\operatorname{Rfd}_{R_{\mathfrak{m}}} M_{\mathfrak{m}}\right\} \\
& \geq \inf \mathrm{H}(C)-\sup _{\mathfrak{m} \in \operatorname{Max} R}\left\{\operatorname{Rfd}_{R_{\mathfrak{m}}} M_{\mathfrak{m}}\right\} \\
& =\inf \mathrm{H}(C)-\operatorname{Rfd}_{R} M \\
& >-\infty,
\end{aligned}
$$

where the first inequality comes from Theorem 3.2 applied over $R_{\mathfrak{m}}$ to the complex $L=\mathrm{RHom}_{R_{\mathfrak{m}}}\left(M_{\mathfrak{m}}, C_{\mathfrak{m}}\right)$, while the last inequality is given by Theorem 1.1. It now follows from Theorem 2.1 that $M$ is derived $C$-reflexive.

The relations above and A.1 yield the desired bounds on amplitude.

Notes 3.4. The equivalence (i) $\Longleftrightarrow$ (ii) in Theorem 3.3 follows from [Christensen 2000, 2.1.10; 2001, 2.11]; see [Frankild et al. 2009, 3.3]. When $\operatorname{dim} R$ is finite, a weaker form of (iii) $\Longrightarrow$ (i) is proved in [Frankild and Sather-Wagstaff 2007, 2.8]: $\delta_{M_{\mathfrak{m}}}^{C_{\mathfrak{m}}}$ an isomorphism for all $\mathfrak{m} \in \operatorname{Max} R$ implies that $\delta_{M}^{C}$ is one.

When $R$ is Cohen-Macaulay and local each semidualizing complex $C$ satisfies $\operatorname{amp} \mathrm{H}(C)=0$, so it is isomorphic to a shift of a finite module; see [Christensen 2001, 3.4].

\section{Perfect complexes}

Recall that a complex of $R$-modules is said to be perfect if it is isomorphic in $\mathrm{D}(R)$ to a bounded complex of finite projective modules. For ease of reference we collect, with complete proofs, some useful tests for perfection; the equivalence of 
(i) and (ii) is contained in [Christensen 2000, 2.1.10], while the argument that (i) are (iii) are equivalent is modelled on a proof when $M$ is a module, due to Bass and Murthy [Bass and Murthy 1967, 4.5].

Theorem 4.1. For a complex $M$ in $\mathrm{D}_{\mathrm{b}}^{\mathrm{f}}(R)$ the following conditions are equivalent.

(i) $M$ is perfect.

(ii) $\operatorname{RHom}_{R}(M, R)$ is perfect.

(iii) $M_{\mathfrak{m}}$ is perfect in $\mathrm{D}\left(R_{\mathfrak{m}}\right)$ for each $\mathfrak{m} \in \operatorname{Max} R$.

(iii') $P_{M_{\mathfrak{m}}}^{R_{\mathfrak{m}}}(t)$ is a Laurent polynomial for each $\mathfrak{m} \in \operatorname{Max} R$.

(iv) $U^{-1} M$ is perfect in $\mathrm{D}\left(U^{-1} R\right)$ for each multiplicatively closed set $U \subseteq R$.

Proof. (iv) $\Longrightarrow$ (iii). This implication is a tautology.

(iii) $\Longrightarrow$ (i). Choose a resolution $F \stackrel{\simeq}{\rightrightarrows} M$ with each $F^{i}$ finite free and zero for $i \ll 0$. Set $s=\sup \mathrm{H}(F)+1$ and $H=\operatorname{Im}\left(\partial_{s}^{F}\right)$, and note that the complex $\Sigma^{-s} F_{\geqslant s}$ is a free resolution of $H$. Since each $R$-module $\operatorname{Im}\left(\partial_{n}^{F}\right)$ is finite, the subset of primes $\mathfrak{p} \in \operatorname{Spec} R$ with $\operatorname{Im}\left(\partial_{n}^{F}\right)_{\mathfrak{p}}$ projective over $R_{\mathfrak{p}}$ is open. It follows that the set

$$
D_{n}=\left\{\mathfrak{p} \in \operatorname{Spec} R \mid \operatorname{pd}_{R_{\mathfrak{p}}} H_{\mathfrak{p}} \leq n\right\}
$$

is open in Spec $R$ for every $n \geq 0$. One has $D_{n} \subseteq D_{n+1}$ for $n \geq 0$, and the hypothesis means $\bigcup_{n \geqslant 0} D_{n}=\operatorname{Spec} R$. As $\operatorname{Spec} R$ is noetherian, it follows that $D_{p}=\operatorname{Spec} R$ holds for some $p \geq 0$, so that $\operatorname{Im}\left(\partial_{s+p}^{F}\right)$ is projective. Taking $E_{n}=0$ for $n>s+p$, $E_{s+p}=\operatorname{Im}\left(\partial_{s+p}^{F}\right)$, and $E_{n}=F_{n}$ for $n<s+p$ one gets a perfect subcomplex $E$ of $F$. The inclusion $E \rightarrow F$ is a quasiisomorphism, so $F$ is perfect.

(i) $\Longrightarrow$ (iv) and (i) $\Longrightarrow$ (ii). In $\mathrm{D}(R)$ one has $M \simeq F$ with $F$ a bounded complex of finite projective $R$-modules. This implies isomorphisms $U^{-1} M \simeq U^{-1} F$ in $\mathrm{D}\left(U^{-1} R\right)$ and $\mathrm{RHom}_{R}(M, R) \simeq \mathrm{RHom}_{R}(F, R)$ in $\mathrm{D}(R)$, with bounded complexes of finite projective modules on their right hand sides.

(ii) $\Longrightarrow$ (i). The perfect complex $N=\mathrm{RHom}_{R}(M, R)$ is evidently derived $R$ reflexive, so the implication (ii) $\Longrightarrow$ (i) in Theorem 2.1 applied with $C=R$ gives $M \simeq \operatorname{RHom}_{R}(N, R)$; as we have just seen, $\operatorname{RHom}_{R}(N, R)$ is perfect along with $N$.

(iii) $\Longleftrightarrow\left(\right.$ (iii' $^{\prime}$. We may assume that $R$ is local with maximal ideal $\mathfrak{m}$. By A.4.1, there is an isomorphism $F \simeq M$ in $\mathrm{D}(R)$, with each $F_{n}$ finite free, $\partial(F) \subseteq \mathfrak{m} F$, and $P_{M}^{R}(t)=\sum_{n \in \mathbb{Z}} \operatorname{rank}_{R} F_{n} t^{n}$. Thus, $M$ is perfect if and only if $F_{n}=0$ holds for all $n \gg 0$; that is, if and only if $P_{M}^{R}(t)$ is a Laurent polynomial.

The following elementary property of perfect complexes is well known:

4.2. If $M$ and $N$ are perfect complexes, then so are $M \otimes_{R}^{\mathrm{L}} N$ and $\operatorname{RHom}_{R}(M, N)$.

To prove a converse we use a version of a result from [Foxby and Iyengar 2003], which incorporates a deep result in commutative algebra, the New Intersection Theorem. 
Theorem 4.3. When $M$ is a perfect complex of $R$-modules and $N$ a complex in $\mathrm{D}^{\mathrm{f}}(R)$ satisfying $\operatorname{Supp}_{R} N \subseteq \operatorname{Supp}_{R} M$, the following inequalities hold:

$$
\begin{aligned}
\sup \mathrm{H}(N) & \leq \sup \mathrm{H}\left(M \otimes_{R}^{\mathrm{L}} N\right)-\inf \mathrm{H}(M), \\
\inf \mathrm{H}(N) & \geq \inf \mathrm{H}\left(M \otimes_{R}^{\mathrm{L}} N\right)-\sup \mathrm{H}(M), \\
\operatorname{amp} \mathrm{H}(N) & \leq \operatorname{amp} \mathrm{H}\left(M \otimes_{R}^{\mathrm{L}} N\right)+\operatorname{amp} \mathrm{H}(M) .
\end{aligned}
$$

If $M \otimes_{R}^{\mathrm{L}} N$ or $\operatorname{RHom}_{R}(M, N)$ is in $\mathrm{D}_{\mathrm{b}}(R)$, then $N$ is in $\mathrm{D}_{\mathrm{b}}^{\mathrm{f}}(R)$.

Proof. For each $\mathfrak{p}$ in $\operatorname{Supp}_{R} N$ the complex $M_{\mathfrak{p}}$ is perfect and nonzero in $\mathrm{D}\left(R_{\mathfrak{p}}\right)$.

The second link in the following chain comes from [Foxby and Iyengar 2003, 3.1], and the rest are standard:

$$
\begin{aligned}
\sup \mathrm{H}(N)_{\mathfrak{p}} & =\sup \mathrm{H}\left(N_{\mathfrak{p}}\right) \\
& \leq \sup \mathrm{H}\left(M_{\mathfrak{p}} \otimes_{R_{\mathfrak{p}}}^{\mathrm{L}} N_{\mathfrak{p}}\right)-\inf \mathrm{H}\left(M_{\mathfrak{p}}\right) \\
& =\sup \mathrm{H}\left(M \otimes_{R}^{\mathrm{L}} N\right)_{\mathfrak{p}}-\inf \mathrm{H}(M)_{\mathfrak{p}} \\
& \leq \sup \mathrm{H}\left(M \otimes_{R}^{\mathrm{L}} N\right)-\inf \mathrm{H}(M) .
\end{aligned}
$$

The first inequality follows, as one has $\sup \mathrm{H}(N)=\sup _{\mathfrak{p} \in \operatorname{Supp} N}\left\{\sup \mathrm{H}(N)_{\mathfrak{p}}\right\}$.

Lemma A.4.3 gives the second link in the next chain, and the rest are standard:

$$
\begin{aligned}
\inf \mathrm{H}(N)_{\mathfrak{p}} & =\inf \mathrm{H}\left(N_{\mathfrak{p}}\right) \\
& =\inf \mathrm{H}\left(M_{\mathfrak{p}} \otimes_{R_{\mathfrak{p}}}^{\mathrm{L}} N_{\mathfrak{p}}\right)-\inf \mathrm{H}(M)_{\mathfrak{p}} \\
& =\inf \mathrm{H}\left(M \otimes_{R}^{\mathrm{L}} N\right)_{\mathfrak{p}}-\inf \mathrm{H}(M)_{\mathfrak{p}} \\
& \geq \inf \mathrm{H}\left(M \otimes_{R}^{\mathrm{L}} N\right)-\sup \mathrm{H}(M) .
\end{aligned}
$$

The second inequality follows, as one has $\inf \mathrm{H}(N)=\inf _{\mathfrak{p} \in \operatorname{Supp} N}\left\{\inf \mathrm{H}(N)_{\mathfrak{p}}\right\}$.

The first two inequalities imply the third one, which contains the assertion concerning $M \otimes_{R}^{\mathrm{L}} N$. In turn, it implies the assertion concerning $\operatorname{RHom}_{R}(M, N)$, because the complex $\operatorname{RHom}_{R}(M, R)$ is perfect along with $M$, one has

$$
\operatorname{Supp}_{R} N \subseteq \operatorname{Supp}_{R} M=\operatorname{Supp}_{R} \operatorname{RHom}_{R}(M, R)
$$

due to A.6, and there is a canonical isomorphism

$$
\operatorname{RHom}_{R}(M, R) \otimes_{R}^{\mathrm{L}} N \simeq \operatorname{RHom}_{R}(M, N) .
$$

Corollary 4.4. Let $M$ be a perfect complex and $N$ a complex in $\mathrm{D}^{\mathrm{f}}(R)$ satisfying $\operatorname{Supp}_{R} N \subseteq \operatorname{Supp}_{R} M$. If $M \otimes_{R}^{\mathrm{L}} N$ or $\operatorname{RHom}_{R}(M, N)$ is perfect, then so is $N$.

Proof. Suppose $M \otimes_{R}^{\mathrm{L}} N$ is perfect; then $N \in \mathrm{D}_{\mathrm{b}}^{\mathrm{f}}(R)$ holds, by Theorem 4.3. For each $\mathfrak{m} \in \operatorname{Max} R$, Theorem 4.1 and Lemma A.4.3 imply that $P_{M_{\mathfrak{m}}}^{R_{\mathfrak{m}}}(t) P_{N_{\mathfrak{m}}}^{R_{\mathfrak{m}}}(t)$ is a 
Laurent polynomial, and hence so is $P_{N_{\mathfrak{m}}}^{R_{\mathfrak{m}}}(t)$. Another application of Theorem 4.1 now shows that $N$ is perfect.

The statement about $\operatorname{RHom}_{R}(M, N)$ follows from the one concerning derived tensor products, by using the argument for the last assertion of the theorem.

Next we establish a stability property of derived reflexivity. The forward implication is well known; see, for instance, [Christensen 2001, 3.17].

Theorem 4.5. Let $M$ be a perfect complex and $C$ a complex in $\mathrm{D}_{-}^{\mathrm{f}}(R)$.

If $N$ in $\mathrm{D}(R)$ is derived $C$-reflexive, then so is $M \otimes_{R}^{\mathrm{L}} N$.

Conversely, for $N$ in $\mathrm{D}^{\mathrm{f}}(R)$ satisfying $\operatorname{Supp}_{R} N \subseteq \operatorname{Supp}_{R} M$, if $M \otimes_{R}^{\mathrm{L}} N$ is derived $C$-reflexive, then so is $N$.

Proof. We may assume that $M$ is a bounded complex of finite projective $R$ modules.

Note that derived $C$-reflexivity is preserved by translation, direct sums, and direct summands, and that if two of the complexes in some exact triangle are derived $C$-reflexive, then so is the third. A standard induction on the number of nonzero components of $M$ shows that when $N$ is derived $C$-reflexive, so is $M \otimes_{R} N$.

Assume that $M \otimes_{R}^{\mathrm{L}} N$ is derived $C$-reflexive and $\operatorname{Supp}_{R} N \subseteq \operatorname{Supp}_{R} M$ holds. Theorem 4.3 gives $N \in \mathrm{D}_{\mathrm{b}}^{\mathrm{f}}(R)$. For the complex $M^{*}=\operatorname{RHom}_{R}(M, R)$ and the functor $\mathrm{h}(-)=\mathrm{RHom}_{R}(-, C)$, in $\mathrm{D}(R)$ there is a natural isomorphism

$$
M^{*} \otimes_{R}^{\mathrm{L}} \mathrm{h}(N) \simeq \mathrm{h}\left(M \otimes_{R}^{\mathrm{L}} N\right) .
$$

Now $\mathrm{h}(N)$ is in $\mathrm{D}^{\mathrm{f}}(R)$ because $N$ is in $\mathrm{D}_{\mathrm{b}}^{\mathrm{f}}(R)$ and $C$ is in $\mathrm{D}_{-}^{\mathrm{f}}(R)$, by [Hartshorne 1966 , p. 92, 3.3]. Since $M$ is perfect, one has that

$$
\operatorname{Supp}_{R} \mathrm{~h}(N) \subseteq \operatorname{Supp}_{R} N \subseteq \operatorname{Supp}_{R} M=\operatorname{Supp}_{R} M^{*},
$$

so Theorem 4.3 gives $\mathrm{h}(N) \in \mathrm{D}_{\mathrm{b}}^{\mathrm{f}}(R)$. Thus, $\mathrm{h}^{2}(N)$ is in $\mathrm{D}^{\mathrm{f}}(R)$, so the isomorphism

$$
M \otimes_{R}^{\mathrm{L}} \mathrm{h}^{2}(N) \simeq \mathrm{h}^{2}\left(M \otimes_{R}^{\mathrm{L}} N\right)
$$

and Theorem 4.3 yield $\mathrm{h}^{2}(N) \in \mathrm{D}_{\mathrm{b}}^{\mathrm{f}}(R)$. Forming an exact triangle

$$
N \stackrel{\delta_{N}^{C}}{\longrightarrow} \mathrm{h}^{2}(N) \longrightarrow W \longrightarrow
$$

one then gets $W \in \mathrm{D}_{\mathrm{b}}^{\mathrm{f}}(R)$ and $\operatorname{Supp}_{R} W \subseteq \operatorname{Supp}_{R} N$.

In the induced exact triangle

$$
M \otimes_{R}^{\mathrm{L}} N \stackrel{M \otimes_{R}^{\mathrm{L}} \delta_{N}^{C}}{\longrightarrow} M \otimes_{R}^{\mathrm{L}} \mathrm{h}^{2}(N) \longrightarrow M \otimes_{R}^{\mathrm{L}} W \longrightarrow
$$

the morphism $M \otimes_{R}^{\mathrm{L}} \delta_{N}^{C}$ is an isomorphism, as its composition with the isomorphism in (4.5.1) is equal to $\delta_{M \otimes_{R}^{L} N}^{C}$, which is an isomorphism by hypothesis. Thus, we 
obtain

$$
M \otimes_{R}^{\mathrm{L}} W=0 \quad \text { in } \mathrm{D}(R),
$$

hence $W=0$ by A.6, so $\delta_{N}^{C}$ is a isomorphism.

Sometimes, the perfection of a complex can be deduced from its homology.

Let $H$ be a graded $R$-module. We say that $H$ is (finite) graded projective if it is bounded and for each $i \in \mathbb{Z}$ the $R$-module $H_{i}$ is (finite) projective.

4.6. If $M$ is a complex of $R$-modules such that $\mathrm{H}(M)$ is projective, then $M \simeq \mathrm{H}(M)$ in $\mathrm{D}(R)$, by [Avramov et al. 2010a, 1.6]. Thus when $\mathrm{H}(M)$ is in addition finite, $M$ is perfect.

We recall some facts about projectivity and idempotents; see also [Avramov and Iyengar 2008, 2.5].

4.7. Let $H$ be a finite graded projective $R$-module.

The $R_{\mathfrak{p}}$-module $\left(H_{i}\right)_{\mathfrak{p}}$ then is finite free for every $\mathfrak{p} \in \operatorname{Spec} R$ and every $i \in \mathbb{Z}$, and one has $\left(H_{i}\right)_{\mathfrak{p}}=0$ for almost all $i$, so $H$ defines a function

$$
r_{H}: \operatorname{Spec} R \rightarrow \mathbb{N} \quad \text { given by } r_{H}(\mathfrak{p})=\sum_{i \in \mathbb{Z}} \operatorname{rank}_{R_{\mathfrak{p}}}\left(H_{i}\right)_{\mathfrak{p}}
$$

One has $r_{H}(\mathfrak{p})=\operatorname{rank}_{R_{\mathfrak{p}}}\left(\bigoplus_{i \in \mathbb{Z}} H_{i}\right)_{\mathfrak{p}}$; since the $R$-module $\bigoplus_{i \in \mathbb{Z}} H_{i}$ is finite projective, $r_{H}$ is constant on each connected component of Spec $R$.

We say that $H$ has rank $d$, and write $\operatorname{rank}_{R} H=d$, if $r_{H}(\mathfrak{p})=d$ holds for every $\mathfrak{p} \in \operatorname{Spec} R$. We say that $H$ is invertible if it is graded projective of rank 1 .

4.8. Let $\left\{a_{1}, \ldots, a_{s}\right\}$ be the (unique) complete set of orthogonal primitive idempotents of $R$. The open subsets $D_{a_{i}}=\left\{\mathfrak{p} \in \operatorname{Spec} R \mid \mathfrak{p} \not \supset a_{i}\right\}$ for $i=1, \ldots, s$ are then the distinct connected components of Spec $R$.

An element $a$ of $R$ is idempotent if and only if $a=a_{i_{1}}+\cdots+a_{i_{r}}$ with indices $1 \leq i_{1}<\cdots<i_{r} \leq s$; this sequence of indices is uniquely determined.

Let $a$ be an idempotent and $-{ }_{a}$ denote localization at the multiplicatively closed set $\{1, a\}$ of $R$. For all $M$ and $N$ in $\mathrm{D}(R)$ there are canonical isomorphisms

$$
\begin{gathered}
M \simeq M_{a} \oplus M_{1-a} \text { and } \\
\operatorname{RHom}_{R}\left(M_{a}, N\right) \simeq \operatorname{RHom}_{R}\left(M_{a}, N_{a}\right) \simeq \operatorname{RHom}_{R}\left(M, N_{a}\right) .
\end{gathered}
$$

In particular, when $M$ is in $\mathrm{D}_{\mathrm{b}}^{\mathrm{f}}(R)$ so is $M_{a}$, and there is an isomorphism $M \simeq M_{a}$ in $\mathrm{D}(R)$ if and only if one has $\operatorname{Supp}_{R} M=D_{a}$.

Every graded $R$-module $L$ has a canonical decomposition $L=\bigoplus_{i=1}^{s} L_{a_{i}}$.

The next result sounds — for the first time in this paper — the theme of rigidity. 
Theorem 4.9. Let $L$ be a complex in $\mathrm{D}_{-}^{\mathrm{f}}(R)$.

If $M$ in $\mathrm{D}_{\mathrm{b}}^{\mathrm{f}}(R)$ satisfies $\operatorname{Supp}_{R} M \supseteq \operatorname{Supp}_{R} L$ and there is an isomorphism

$$
M \simeq \operatorname{RHom}_{R}(L, M) \quad \text { or } \quad M \simeq L \otimes_{R}^{\mathrm{L}} M,
$$

then for some idempotent $a$ in $R$ the $R_{a}$-module $\mathrm{H}_{0}(L)_{a}$ is invertible and one has

$$
L \simeq \mathrm{H}_{0}(L) \simeq \mathrm{H}_{0}(L)_{a} \simeq L_{a} \quad \text { in } \mathrm{D}(R) .
$$

The element a is determined by either one of the following equalities:

$$
\operatorname{Supp}_{R} M=\{\mathfrak{p} \in \operatorname{Spec} R \mid \mathfrak{p} \not \supset a\}=\operatorname{Supp}_{R} L .
$$

Proof. If $\mathrm{H}(M)=0$, then the hypotheses imply $\operatorname{Supp}_{R} L=\varnothing$, so $a=0$ is the desired idempotent. For the rest of the proof we assume $\mathrm{H}(M) \neq 0$.

If $M \simeq \operatorname{RHom}_{R}(L, M)$ holds and $\mathfrak{m}$ is in $\operatorname{Max} R \cap \operatorname{Supp}_{R} M$, then Lemma A.5.3 shows that $L_{\mathfrak{m}}$ is in $\mathrm{D}_{+}^{\mathrm{f}}\left(R_{\mathfrak{m}}\right)$ and gives the second equality below:

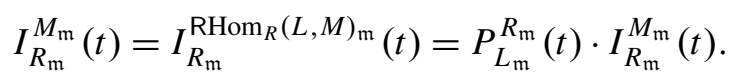

As $I_{R_{\mathfrak{m}}}^{M_{\mathfrak{m}}}(t) \neq 0$ by A.5.2, this gives $P_{L_{\mathfrak{m}}}^{R_{\mathfrak{m}}}(t)=1$, and hence $L_{\mathfrak{m}} \simeq R_{\mathfrak{m}}$ by A.4.1. Thus, for every $\mathfrak{p} \in \operatorname{Supp}_{R} M$ one has $L_{\mathfrak{p}} \simeq R_{\mathfrak{p}}$, which yields

$$
\operatorname{Supp}_{R} M=\operatorname{Supp}_{R} L=\operatorname{Supp}_{R} \mathrm{H}_{0}(L)
$$

and shows the $R$-module $\mathrm{H}_{0}(L)$ is projective with $\operatorname{rank}_{R_{\mathfrak{p}}} \mathrm{H}_{0}(L)_{\mathfrak{p}}=1$ for each $\mathfrak{p} \in \operatorname{Supp}_{R} \mathrm{H}_{0}(L)$. The rank of a projective module is constant on connected components of $\operatorname{Spec} R$, and therefore $\operatorname{Supp}_{R} \mathrm{H}_{0}(L)$ is a union of such components, whence, by 4.8 , there is a unique idempotent $a \in R$, such that

$$
\operatorname{Supp}_{R} \mathrm{H}_{0}(L)=\{\mathfrak{p} \in \operatorname{Spec} R \mid \mathfrak{p} \not \ngtr a\},
$$

and the graded $R_{a}$-module $\mathrm{H}(L)_{a}$ is invertible. The preceding discussion, 4.8, and 4.6 give isomorphisms $L \simeq \mathrm{H}_{0}(L) \simeq \mathrm{H}_{0}(L)_{a} \simeq L_{a}$ in $\mathrm{D}(R)$.

A similar argument, using Lemma A.4.3 and A.4.2, applies if $M \simeq L \otimes_{R}^{L} M$.

\section{Invertible complexes}

We say that a complex in $\mathrm{D}(R)$ is invertible if it is semidualizing and perfect.

The following canonical morphisms, defined for all $L, M$, and $N$ in $\mathrm{D}(R)$, play a role in characterizing invertible complexes and in using them: Evaluation

$$
\mathrm{RHom}_{R}(L, N) \otimes_{R}^{\mathrm{L}} L \longrightarrow N
$$


is induced by the chain map $\lambda \otimes l \mapsto \lambda(l)$. Tensor-evaluation is the composition

$$
\begin{aligned}
\operatorname{RHom}_{R}\left(M \otimes_{R}^{\mathrm{L}} L, N\right) \otimes_{R}^{\mathrm{L}} L & \stackrel{\simeq}{ } \operatorname{RHom}_{R}\left(L \otimes_{R}^{\mathrm{L}} M, N\right) \otimes_{R}^{\mathrm{L}} L \\
& \stackrel{\simeq}{\longrightarrow} \operatorname{RHom}_{R}\left(L, \operatorname{RHom}_{R}(M, N)\right) \otimes_{R}^{\mathrm{L}} L \\
& \longrightarrow \operatorname{RHom}_{R}(M, N),
\end{aligned}
$$

where the isomorphisms are canonical and the last arrow is given by evaluation.

The equivalence of conditions (i) and ( $\left.i^{\prime}\right)$ in the result below shows that for complexes with zero differential, invertibility agrees with the notion in 4.7. Invertible complexes coincide with the tilting complexes of Frankild, Sather-Wagstaff, and Taylor, see [Frankild et al. 2009, 4.7], where some of the following equivalences are proved.

Proposition 5.1. For $L \in \mathrm{D}_{\mathrm{b}}^{\mathrm{f}}(R)$ the following conditions are equivalent.

(i) $L$ is invertible in $\mathrm{D}(R)$.

(i') $\mathrm{H}(L)$ is an invertible graded $R$-module.

(ii) $\mathrm{RHom}_{R}(L, R)$ is invertible in $\mathrm{D}(R)$.

(ii') $\operatorname{Ext}_{R}(L, R)$ is an invertible graded $R$-module.

(iii) For each $\mathfrak{p} \in \operatorname{Spec} R$ one has $L_{\mathfrak{p}} \simeq \Sigma^{r(\mathfrak{p})} R_{\mathfrak{p}}$ in $\mathrm{D}\left(R_{\mathfrak{p}}\right)$ for some $r(\mathfrak{p}) \in \mathbb{Z}$.

(iii') For each $\mathfrak{m} \in \operatorname{Max} R$ one has $P_{L_{\mathfrak{m}}}^{R_{\mathfrak{m}}}(t)=t^{r(\mathfrak{m})}$ for some $r(\mathfrak{m}) \in \mathbb{Z}$.

(iv) $U^{-1} L$ is invertible in $\mathrm{D}\left(U^{-1} R\right)$ for each multiplicatively closed set $U \subseteq R$.

(v) For some $N$ in $\mathrm{D}^{\mathrm{f}}(R)$ there is an isomorphism $N \otimes_{R}^{\mathrm{L}} L \simeq R$.

(vi) For each $N$ in $\mathrm{D}(R)$ the evaluation map (5.0.1) is an isomorphism.

(vi') For all $M, N$ in $\mathrm{D}(R)$ the tensor-evaluation map (5.0.2) is an isomorphism.

Proof. (i) $\Longleftrightarrow$ (iv). This follows from Proposition 3.1 and Theorem 4.1.

(i) $\Longrightarrow$ (vi). The first two isomorphisms below hold because $L$ is perfect:

$\operatorname{RHom}_{R}(L, N) \otimes_{R}^{\mathrm{L}} L \simeq \operatorname{RHom}_{R}\left(L, L \otimes_{R}^{\mathrm{L}} N\right) \simeq \operatorname{RHom}_{R}(L, L) \otimes_{R}^{\mathrm{L}} N \simeq N$.

The third one holds because $L$ is semidualizing.

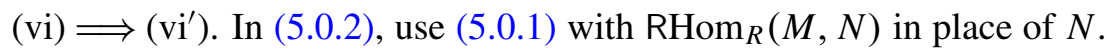

(vi') $\Longrightarrow$ (vi). Set $M=R$ in (5.0.2).

(vi) $\Longrightarrow(\mathrm{v})$. Setting $N=R$ one gets an isomorphism $\operatorname{RHom}_{R}(L, R) \otimes_{R}^{L} L \simeq R$. Note that $\operatorname{RHom}_{R}(L, R)$ is in $\mathrm{D}_{-}^{\mathrm{f}}(R)$, since $L$ is in $\mathrm{D}_{\mathrm{b}}^{\mathrm{f}}(R)$.

Condition (v) localizes, and the already-proved equivalence of (i) and (iv) shows that conditions (i) and (ii) can be checked locally. Clearly, the same holds true for conditions (i'), (ii'), (iii'), and (iii). Thus, in order to finish the proof it suffices to show that when $R$ is a local ring there exists a string of implications linking (v) to (i) and passing through the remaining conditions. 
$(\mathrm{v}) \Longrightarrow\left(\right.$ iii' $\left.^{\prime}\right)$. Lemma A.4.3 gives $P_{N}^{R}(t) \cdot P_{L}^{R}(t)=1$. Such an equality of formal Laurent series implies $P_{L}^{R}(t)=t^{r}$ and $P_{N}^{R}(t)=t^{-r}$ for some integer $r$.

(iii' $) \Longrightarrow$ (iii). This follows from A.4.1.

(iii) $\Longrightarrow\left(\mathrm{i}^{\prime}\right)$. This implication is evident.

$\left(\mathrm{i}^{\prime}\right) \Longrightarrow\left(\mathrm{ii}^{\prime}\right)$. As $\mathrm{H}(L)$ is projective one has $L \simeq \mathrm{H}(L)$ in $\mathrm{D}(R)$ (see 4.6), hence

$$
\operatorname{Ext}_{R}(L, R) \cong \operatorname{Ext}_{R}(\mathrm{H}(L), R) \cong \operatorname{Hom}_{R}(\mathrm{H}(L), R)
$$

Now note that the graded module $\operatorname{Hom}_{R}(\mathrm{H}(L), R)$ is invertible because $\mathrm{H}(L)$ is.

$\left(\mathrm{ii}^{\prime}\right) \Longrightarrow$ (ii). Because $\mathrm{H}\left(\operatorname{RHom}_{R}(L, R)\right)$ is projective, 4.6 gives the first isomorphism below; the second one holds (for some $r \in \mathbb{Z}$ ) because $R$ is local:

$$
\operatorname{RHom}_{R}(L, R) \simeq \mathrm{H}\left(\operatorname{RHom}_{R}(L, R)\right)=\operatorname{Ext}_{R}(L, R) \simeq \Sigma^{r} R
$$

(ii) $\Longrightarrow$ (i). The invertible complex $L^{\prime}=\operatorname{RHom}_{R}(L, R)$ is evidently derived $R$-reflexive, so the implication (ii) $\Longrightarrow$ (i) in Theorem 2.1 applies with $C=R$. It gives $L \simeq \operatorname{RHom}_{R}\left(L^{\prime}, R\right)$; now note that $\operatorname{RHom}_{R}\left(L^{\prime}, R\right)$ is invertible along with L.

Recall that $\operatorname{Pic}(R)$ denotes the Picard group of $R$, such that its elements are isomorphism classes of invertible $R$-modules, multiplication is induced by tensor product over $R$, and the class of $\operatorname{Hom}_{R}(L, R)$ is the inverse of that of $L$. A derived version of this construction is given in [Frankild et al. 2009, 4.1] and is recalled below; it coincides with the derived Picard group of $R$ relative to itself, in the sense of Yekutieli [1999, 3.1].

5.2. When $L$ is an invertible complex, we set

$$
L^{-1}=\operatorname{RHom}_{R}(L, R) .
$$

Condition (vi) of Proposition 5.1 gives for each $N \in \mathrm{D}(R)$ an isomorphism

$$
\mathrm{RHom}_{R}(L, N) \simeq L^{-1} \otimes_{R}^{\mathrm{L}} N
$$

In view of 4.6, condition $\left(i^{\prime}\right)$ of Proposition 5.1 implies that the isomorphism classes $[L]$ of invertible complexes $L$ in $\mathrm{D}(R)$ form a set, which we call DPic $(R)$. As derived tensor products are associative and commutative, $\operatorname{DPic}(R)$ carries the natural structure of an abelian group, with unit element $[R]$, and $[L]^{-1}=\left[L^{-1}\right]$. Following [Frankild et al. 2009, 4.3.1], we refer to it as the derived Picard group of $R$.

We say that complexes $M$ and $N$ are derived Picard equivalent if there is an isomorphism $N \simeq L \otimes_{R}^{\mathrm{L}} M$ for some invertible complex $L$.

Clearly, if $N$ and $N^{\prime}$ are complexes in $\mathrm{D}(R)$ which satisfy $L \otimes_{R}^{\mathrm{L}} N \simeq L \otimes_{R}^{\mathrm{L}} N^{\prime}$ or $\operatorname{RHom}_{R}(L, N) \simeq \operatorname{RHom}_{R}\left(L, N^{\prime}\right)$, then $N \simeq N^{\prime}$. 
The derived Picard group of a local ring $R$ is the free abelian group with generator $[\Sigma R$ ]; see [Frankild et al. 2009, 4.3.4]. In general, one has the following description, which is a special case of [Yekutieli 1999, 3.5]. We include a proof, for the sake of completeness.

Proposition 5.3. There exists a canonical isomorphism of abelian groups

$$
\operatorname{DPic}(R) \stackrel{\cong}{\longrightarrow} \prod_{i=1}^{s}\left(\operatorname{Pic}\left(R_{a_{i}}\right) \times \mathbb{Z}\right),
$$

where $\left\{a_{1}, \ldots, a_{s}\right\}$ is the complete set of primitive orthogonal idempotents; see 4.8 .

Proof. By Proposition 5.1, every element of $\operatorname{DPic}(R)$ is equal to $[L]$ for some graded invertible $R$-module $L$. In the canonical decomposition from 4.8 each $R_{a_{i}}$-module $L_{a_{i}}$ is graded invertible. It is indecomposable because $\operatorname{Spec}\left(R_{a_{i}}\right)$ is connected, hence $L_{a_{i}} \cong \Sigma^{n_{i}} L_{i}$ with uniquely determined invertible $R_{a_{i}}$-module $L_{i}$ and $n_{i} \in \mathbb{Z}$. The map $[L] \mapsto\left(\left(\left[L_{1}\right], n_{1}\right), \ldots,\left(\left[L_{s}\right], n_{s}\right)\right)$ gives the desired isomorphism.

Other useful properties of derived Picard group actions are collected in the next two results, which overlap with [Frankild et al. 2009, 4.8]; we include proofs for completeness.

Lemma 5.4. For L invertible, and $C$ and $M$ in $\mathrm{D}_{\mathrm{b}}^{\mathrm{f}}(R)$, the following are equivalent.

(i) $M$ is derived $C$-reflexive.

(ii) $M$ is derived $L \otimes_{R}^{\mathrm{L}} C$-reflexive.

(iii) $L \otimes_{R}^{\mathrm{L}} M$ is derived $C$-reflexive.

Proof. (i) $\Longrightarrow$ (ii). Since $L$ is invertible, the morphism

$$
\vartheta: L \otimes_{R}^{\mathrm{L}} \mathrm{RHom}_{R}(M, C) \rightarrow \operatorname{RHom}_{R}\left(M, L \otimes_{R}^{\mathrm{L}} C\right)
$$

represented by $l \otimes \alpha \mapsto(m \mapsto l \otimes \alpha(m))$, is an isomorphism: It suffices to check the assertion after localizing at each $\mathfrak{p} \in \operatorname{Spec} R$, where it follows from $L_{\mathfrak{p}} \cong$ $R_{\mathfrak{p}}$. In particular, since $\mathrm{RHom}_{R}(M, C)$ is in $\mathrm{D}_{\mathrm{b}}^{\mathrm{f}}(R)$, so is $\operatorname{RHom}_{R}\left(M, L \otimes_{R}^{\mathrm{L}} C\right)$. Furthermore, in $\mathrm{D}(R)$ there is a commutative diagram of canonical morphisms

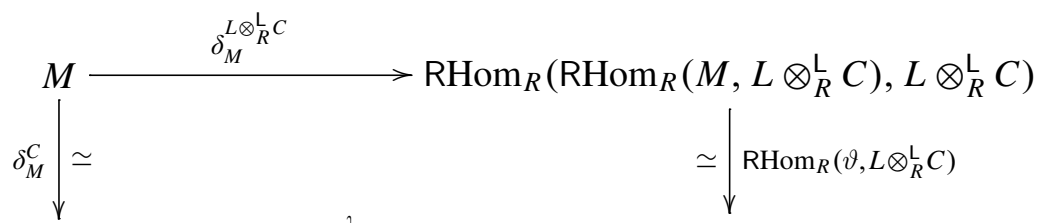

$\mathrm{RHom}_{R}\left(\operatorname{RHom}_{R}(M, C), C\right) \underset{\simeq}{\stackrel{\lambda}{\simeq}} \mathrm{RHom}_{R}\left(L \otimes_{R}^{\mathrm{L}} \operatorname{RHom}_{R}(M, C), L \otimes_{R}^{\mathrm{L}} C\right)$ 
with $\lambda(\alpha)=L \otimes_{R}^{\mathrm{L}} \alpha$, which is an isomorphism, as is readily verified by localization. Thus, $M$ is derived $L \otimes_{R}^{L} C$-reflexive.

(ii) $\Longrightarrow$ (i). The already established implication (i) $\Longrightarrow$ (ii) shows that $M$ is reflexive with respect to $L^{-1} \otimes_{R}^{\mathrm{L}}\left(L \otimes_{R}^{\mathrm{L}} C\right)$, which is isomorphic to $C$.

(i) $\Longleftrightarrow$ (iii) This follows from Theorem 4.5.

From Proposition 3.1 and Lemma 5.4, we obtain:

Lemma 5.5. For $L$ invertible and $C$ in $\mathrm{D}_{\mathrm{b}}^{\mathrm{f}}(R)$ the following are equivalent.

(i) $C$ is semidualizing.

(ii) $L \otimes_{R}^{\mathrm{L}} C$ is semidualizing.

(iii) $L$ is derived $C$-reflexive.

Invertible complexes are used in [Frankild et al. 2009, 5.1] to characterize mutual reflexivity of a pair of semidualizing complexes. The next theorem is fundamentally different, in that the semidualizing property is part of its conclusions, not of its hypotheses.

Theorem 5.6. For $B$ and $C$ in $\mathrm{D}_{\mathrm{b}}^{\mathrm{f}}(R)$ the following conditions are equivalent.

(i) $B$ is derived $C$-reflexive, $C$ is derived $B$-reflexive, and $\operatorname{Supp}_{R} B=\operatorname{Spec} R$.

(ii) $B$ is semidualizing, $\operatorname{RHom}_{R}(B, C)$ is invertible, and the evaluation map

$$
\operatorname{RHom}_{R}(B, C) \otimes_{R}^{\mathrm{L}} B \rightarrow C
$$

is an isomorphism in $\mathrm{D}(R)$.

(iii) $B$ and $C$ are semidualizing and derived Picard equivalent.

Proof. (i) $\Longrightarrow$ (ii). The hypotheses pass to localizations and, by Propositions 3.1 and 5.1, the conclusions can be tested locally. We may thus assume $R$ is local.

Set $F=\operatorname{RHom}_{R}(B, C)$ and $G=\operatorname{RHom}_{R}(C, B)$. In view of Lemma A.5.3, the isomorphisms $B \simeq \operatorname{RHom}_{R}(F, C)$ and $C \simeq \operatorname{RHom}_{R}(G, B)$ yield

$$
I_{R}^{B}(t)=P_{F}^{R}(t) \cdot I_{R}^{C}(t) \quad \text { and } \quad I_{R}^{C}(t)=P_{G}^{R}(t) \cdot I_{R}^{B}(t) .
$$

As $I_{R}^{B}(t) \neq 0$ holds (see A.5.2) these equalities imply $P_{F}^{R}(t) \cdot P_{G}^{R}(t)=1$, hence $P_{F}^{R}(t)=t^{r}$ holds for some $r$. Proposition 5.1 now gives $F \simeq \Sigma^{r} R$, so one gets

$$
B \simeq \operatorname{RHom}_{R}(F, C) \simeq \operatorname{RHom}_{R}\left(\Sigma^{r} R, C\right) \simeq \Sigma^{-r} C .
$$

Thus, $B$ is derived $B$-reflexive, hence semidualizing by Proposition 3.1. A direct verification shows that the following evaluation map is an isomorphism:

$$
\mathrm{RHom}_{R}\left(\Sigma^{-r} C, C\right) \otimes_{R}^{\mathrm{L}} \Sigma^{-r} C \rightarrow C .
$$

(ii) $\Longrightarrow$ (iii) Lemma 5.5 shows that $C$ is semidualizing; the rest is clear. 
(iii) $\Longrightarrow$ (i). Proposition 3.1 shows that $B$ satisfies $\operatorname{Supp}_{R} B=\operatorname{Spec} R$ and is derived $B$-reflexive. From Lemma 5.4 we then see that $B$ is derived $C$-reflexive. A second loop, this time starting from $C$, shows that $C$ is derived $B$-reflexive.

Taking $B=R$ one recovers a result contained in [Christensen 2001, 8.3].

Corollary 5.7. A complex in $\mathrm{D}(R)$ is invertible if and only if it is semidualizing and derived $R$-reflexive.

\section{Duality}

We say that a contravariant $R$-linear exact functor d: $\mathrm{D}(R) \rightarrow \mathrm{D}(R)$ is a duality on a subcategory $\mathrm{A}$ of $\mathrm{D}(R)$ if it satisfies $\mathrm{d}(\mathrm{A}) \subseteq \mathrm{A}$ and $\left.\mathrm{d}^{2}\right|_{\mathrm{A}}$ is isomorphic to $\mathrm{id}^{\mathrm{A}}$.

In this section we link dualities on subcategories of $\mathrm{D}_{\mathrm{b}}^{\mathrm{f}}(R)$ to semidualizing complexes. In the 'extremal' cases, when the subcategory equals $D_{\mathrm{b}}^{\mathrm{f}}(R)$ itself or when the semidualizing complex is the module $R$, we recover a number of known results and answer some open questions.

6.1. Reflexive subcategories. For each complex $C$ in $\mathrm{D}(R)$, set

$$
\mathrm{h}_{C}=\mathrm{RHom}_{R}(-, C): \mathrm{D}(R) \longrightarrow \mathrm{D}(R) \text {. }
$$

The reflexive subcategory of $C$ is the full subcategory of $\mathrm{D}(R)$ defined by

$$
\mathrm{R}_{C}=\left\{M \in \mathrm{D}_{\mathrm{b}}^{\mathrm{f}}(R) \mid M \simeq \mathrm{h}_{C}^{2}(M)\right\}
$$

By Proposition 2.3, the functor $\mathrm{h}_{C}$ is a duality on $\mathrm{R}_{C}$ provided $\mathrm{h}_{C}\left(\mathrm{R}_{C}\right) \subseteq \mathrm{R}_{C}$ holds. We note that, under an additional condition, such a $C$ has to be semidualizing.

Proposition 6.1.1. Let $\mathrm{d}$ be a duality on a subcategory $\mathrm{A}$ of $\mathrm{D}_{\mathrm{b}}^{\mathrm{f}}(R)$.

If $\mathrm{A}$ contains $R$, then the complex $C=\mathrm{d}(R)$ is semidualizing and $\mathrm{A}$ is contained in $\mathrm{R}_{C}$; furthermore, for each $R$-module $M$ in $\mathrm{A}$ there is an isomorphism

$$
M \simeq \operatorname{RHom}_{R}(\mathrm{~d}(M), C)
$$

Proof. Let $M$ be an $R$-module. For each $n \in \mathbb{Z}$ one then has isomorphisms

$$
\begin{aligned}
\operatorname{Ext}_{R}^{n}(\mathrm{~d}(M), C) & \cong \operatorname{Hom}_{\mathrm{D}(R)}\left(\mathrm{d}(M), \Sigma^{n} C\right) \\
& \cong \operatorname{Hom}_{\mathrm{D}(R)}\left(R, \Sigma^{n} \mathrm{~d}^{2}(M)\right) \\
& \cong \operatorname{Hom}_{\mathrm{D}(R)}\left(R, \Sigma^{n} M\right) \\
& \cong \operatorname{Ext}_{R}^{n}(R, M) \\
& \cong \begin{cases}M & \text { for } n=0 ; \\
0 & \text { for } n \neq 0 .\end{cases}
\end{aligned}
$$

It follows that $\mathrm{RHom}_{R}(\mathrm{~d}(M), C)$ is isomorphic to $M$ in $\mathrm{D}(R)$. For $M=R$ this yields $\mathrm{RHom}_{R}(C, C) \simeq R$, so $C$ is semidualizing by Proposition 3.1 . 
Next we show that semidualizing complexes do give rise to dualities and that, furthermore, they are determined by their reflexive subcategories.

Theorem 6.1.2. Let $C$ be a semidualizing complex for $R$.

The functor $\mathrm{h}_{C}$ is a duality on $\mathrm{R}_{C}$, the natural transformation $\delta^{C}: \mathrm{id} \rightarrow \mathrm{h}_{C}^{2}$ restricts to an isomorphism of functors on $\mathrm{R}_{C}$, and $R$ is in $\mathrm{R}_{C}$.

$A$ complex $B$ in $\mathrm{D}_{\mathrm{b}}^{\mathrm{f}}(R)$ satisfies $\mathrm{R}_{B}=\mathrm{R}_{C}$ if and only if $B$ is derived Picard equivalent to $C$ (in which case $B$ is semidualizing).

Proof. Theorem 3.3 implies that $\mathrm{h}_{C}$ takes values in $\mathrm{R}_{C}$ and that $\delta^{C}$ restricts to an isomorphism on $\mathrm{R}_{C}$, while Proposition 3.1 shows that $R$ and $C$ are in $\mathrm{R}_{C}$.

The last assertion results from Theorem 5.6.

The preceding results raise the question of whether every duality functor on a subcategory of $\mathrm{D}_{\mathrm{b}}^{\mathrm{f}}(R)$ is representable on its reflexive subcategory.

6.2. Dualizing complexes. Let $D$ be a complex in $\mathrm{D}(R)$.

Recall that $D$ is said to be dualizing for $R$ if it is semidualizing and of finite injective dimension. If $D$ is dualizing, then $\mathrm{R}_{D}=\mathrm{D}_{\mathrm{b}}^{\mathrm{f}}(R)$; see [Hartshorne 1966, p. 258, 2.1].

In the language of Hartshorne [1966, p. 286], the complex $D$ is pointwise $d u$ alizing for $R$ if it is in $D_{-}^{f}(R)$ and the complex $D_{\mathfrak{p}}$ is dualizing for $R_{\mathfrak{p}}$ for each $\mathfrak{p} \in \operatorname{Spec} R$. When in addition $D$ is in $\mathrm{D}_{\mathrm{b}}^{\mathrm{f}}(R)$, we say that it is strongly pointwise dualizing; this terminology is due to Gabber; see [Conrad 2000, p. 120 ], also for discussion on why the latter concept is the more appropriate one.

For a different treatment of dualizing complexes, see Neeman [2008].

The next result is classical; see [Hartshorne 1966, p. 283, 7.2; p. 286, Remark $1 ;$ p. 288, 8.2].

6.2.1. Let $D$ be a complex in $\mathrm{D}_{\mathrm{b}}^{\mathrm{f}}(R)$. The complex $D$ is dualizing if and only if it is pointwise dualizing and $\operatorname{dim} R$ is finite.

The equivalence of conditions (i) and (ii) in the next result is due to Gabber; see [Conrad 2000, 3.1.5]. Traces of his argument can be found in our proof, as it refers to Theorem 3.3, and thus depends on Theorem 1.1.

Theorem 6.2.2. For $D$ in $\mathrm{D}(R)$ the following conditions are equivalent.

(i) $D$ is strongly pointwise dualizing for $R$.

(ii) $\mathrm{h}_{D}$ is a duality on $\mathrm{D}_{\mathrm{b}}^{\mathrm{f}}(R)$.

(iii) $D$ is in $\mathrm{D}_{\mathrm{b}}^{\mathrm{f}}(R)$, and for each $\mathfrak{m} \in \operatorname{Max} R$ and finite $R$-module $M$, one has

$$
M_{\mathfrak{m}} \simeq \operatorname{RHom}_{R_{\mathfrak{m}}}\left(\operatorname{RHom}_{R_{\mathfrak{m}}}\left(M_{\mathfrak{m}}, D_{\mathfrak{m}}\right), D_{\mathfrak{m}}\right) \quad \text { in } \mathrm{D}\left(R_{\mathfrak{m}}\right) .
$$


Proof. (i) $\Longrightarrow$ (iii). By definition, $D \in \mathrm{D}_{\mathrm{b}}^{\mathrm{f}}(R)$ and $D_{\mathfrak{m}}$ is dualizing for $R_{\mathfrak{m}}$. Moreover, it is clear that $M_{\mathfrak{m}} \in \mathrm{D}_{\mathrm{b}}^{\mathrm{f}}\left(R_{\mathfrak{m}}\right)=\mathrm{R}_{D_{\mathfrak{m}}}$.

(iii) $\Longrightarrow$ (i). Let $\mathfrak{m}$ be a maximal ideal of $R$. For $M_{\mathfrak{m}}=R_{\mathfrak{m}}$ the hypothesis implies that $D_{\mathfrak{m}}$ is semidualizing; see Proposition 3.1. For $M=R / \mathfrak{m}$ it implies, by the first part of Lemma A.5.3, that $\operatorname{RHom}_{R_{\mathfrak{m}}}\left(R_{\mathfrak{m}} / \mathfrak{m} R_{\mathfrak{m}}, D_{\mathfrak{m}}\right) \in \mathrm{D}_{\mathrm{b}}^{\mathrm{f}}\left(R_{\mathfrak{m}}\right)$; this means that $D_{\mathfrak{m}}$ has finite injective dimension over $R_{\mathfrak{m}}$; see A.5.1. Localization shows that $D_{\mathfrak{p}}$ has the corresponding properties for every prime ideal $\mathfrak{p}$ of $R$, contained in $\mathfrak{m}$.

(iii) $\Longleftrightarrow$ (ii). The complex $D$ is semidualizing — by Proposition 3.1 if (iii) holds, by Proposition 6.1.1 if (ii) holds; so the equivalence results from Theorem 3.3.

Corollary 6.2.3. The ring $R$ is Gorenstein if and only if the complex $R$ is strongly pointwise dualizing, if and only if each complex in $\mathrm{D}_{\mathrm{b}}^{\mathrm{f}}(R)$ is derived $R$-reflexive.

Proof. For arbitrary $R$ and $\mathfrak{p} \in \operatorname{Spec} R$, the complex $R_{\mathfrak{p}}$ is semidualizing for $R_{\mathfrak{p}}$. Thus, the first two conditions are equivalent because - by definition - the ring $R$ is Gorenstein if and only if $R_{\mathfrak{p}}$ has a finite injective resolution as a module over itself for each $\mathfrak{p}$. The second and third conditions are equivalent by Theorem 6.2.2.

Given a homomorphism $R \rightarrow S$ of rings, recall that $\mathrm{RHom}_{R}(S,-)$ is a functor from $\mathrm{D}(R)$ to $\mathrm{D}(S)$. The next result is classical; see [Hartshorne 1966, p. 260, 2.4].

Corollary 6.2.4. If $R \rightarrow S$ is a finite homomorphism of rings and $D \in \mathrm{D}_{\mathrm{b}}^{\mathrm{f}}(R)$ is pointwise dualizing for $R$, then $\operatorname{RHom}_{R}(S, D)$ is pointwise dualizing for $S$.

Proof. Set $D^{\prime}=\operatorname{RHom}_{R}(S, D)$. For each $M$ in $\mathrm{D}_{\mathrm{b}}^{\mathrm{f}}(S)$ one has

$$
\mathrm{RHom}_{R}(M, D) \simeq \operatorname{RHom}_{S}\left(M, D^{\prime}\right) \quad \text { in } \mathrm{D}(S) .
$$

It shows that $\operatorname{RHom}_{S}\left(M, D^{\prime}\right)$ is in $\mathrm{D}_{\mathrm{b}}^{\mathrm{f}}(S)$, and that the restriction of $\mathrm{h}_{D}$ to $\mathrm{D}_{\mathrm{b}}^{\mathrm{f}}(S)$ is equivalent to $\mathrm{h}_{D^{\prime}}$. Theorem 6.2.2 then shows that $D^{\prime}$ is pointwise dualizing.

It follows from Corollaries 6.2.3 and 6.2.4 that if $S$ is a homomorphic image of a Gorenstein ring, then it admits a strongly pointwise dualizing complex. Kawasaki [2002, 1.4] proved that if $S$ has a dualizing complex, then $S$ is a homomorphic image of some Gorenstein ring of finite Krull dimension, so we ask:

Question 6.2.5. Does the existence of a strongly pointwise dualizing complex for $S$ imply that $S$ is a homomorphic image of some Gorenstein ring?

6.3. Finite G-dimension. The category $\mathrm{R}_{R}$ of derived $R$-reflexive complexes contains all perfect complexes, but may be larger. To describe it we use a notion from module theory: An $R$-module $G$ is totally reflexive when it is finite,

$$
\begin{array}{rlrl}
\operatorname{Hom}_{R}\left(\operatorname{Hom}_{R}(G, R), R\right) \cong G & & \text { and } \\
\operatorname{Ext}_{R}^{n}\left(\operatorname{Hom}_{R}(G, R), R\right) & =0=\operatorname{Ext}_{R}^{n}(G, R) & & \text { for all } n \geq 1 .
\end{array}
$$


A complex of $R$-modules is said to have finite G-dimension (for Gorenstein dimension) if it is quasi-isomorphic to a bounded complex of totally reflexive modules. The study of modules of finite $G$-dimension was initiated by Auslander and Bridger [1969]. The next result, taken from [Christensen 2000, 2.3.8], is due to Foxby.

6.3.1. A complex in $\mathrm{D}(R)$ is in $\mathrm{R}_{R}$ if and only if it has finite $G$-dimension.

Theorems 2.1 and 3.3 specialize to:

Theorem 6.3.2. For a complex $M \in \mathrm{D}_{\mathrm{b}}^{\mathrm{f}}(R)$ the following are equivalent.

(i) $M$ is derived $R$-reflexive.

(ii) $\operatorname{RHom}_{R}(M, R)$ is derived $R$-reflexive.

(iii) For each $\mathfrak{m} \in \operatorname{Max} R$ there is an isomorphism

$$
M_{\mathfrak{m}} \simeq \operatorname{RHom}_{R_{\mathfrak{m}}}\left(\mathrm{RHom}_{R_{\mathfrak{m}}}\left(M_{\mathfrak{m}}, R_{\mathfrak{m}}\right), R_{\mathfrak{m}}\right) \quad \text { in } \mathrm{D}\left(R_{\mathfrak{m}}\right) .
$$

(iv) $U^{-1} M$ is derived $U^{-1} R$-reflexive for each multiplicatively closed set $U$.

Combining 6.3.1 and Corollary 6.2.3, we obtain a new proof of a result due to Auslander and Bridger [1969, 4.20] (when $\operatorname{dim} R$ is finite) and to Goto [1982] (in general).

Corollary 6.3.3. The ring $R$ is Gorenstein if and only if every finite $R$-module has finite G-dimension.

It is easy to check that if a complex $M$ has finite $G$-dimension over $R$, then so does the complex of $R_{\mathfrak{p}}$-modules $M_{\mathfrak{p}}$, for any prime ideal $\mathfrak{p}$. Whether the converse holds had been an open question, which we settle as a corollary of 6.3.1 and Theorem 6.3.2:

Corollary 6.3.4. A homologically finite complex $M$ has finite $G$-dimension if (and only if) the complex $M_{\mathfrak{m}}$ has finite G-dimension over $R_{\mathfrak{m}}$ for every $\mathfrak{m} \in \operatorname{Max} R$.

\section{Rigidity}

Over an arbitrary commutative ring, we introduce a concept of rigidity of one complex relative to another, and establish the properties responsible for the name. In Section 8.5 we show how to recover the notion of rigidity for complexes over commutative algebras, defined by Van den Bergh, Yekutieli and Zhang.

Let $C$ be a complex in $\mathrm{D}(R)$. We say that a complex $M$ in $\mathrm{D}(R)$ is $C$-rigid if there exists an isomorphism

$$
\mu: M \stackrel{\simeq}{\rightarrow} \operatorname{RHom}_{R}\left(\operatorname{RHom}_{R}(M, C), M\right) \text { in } \mathrm{D}(R) .
$$

In such a case, we call $\mu$ a $C$-rigidifying isomorphism and $(M, \mu)$ a $C$-rigid pair. 
Example 7.1. Let $C$ be a semidualizing complex. For each idempotent element $a \in R$, using (3.0.1) and 4.8 one obtains a canonical composite isomorphism

$$
\begin{aligned}
& \gamma_{a}: C_{a} \simeq \operatorname{RHom}_{R}\left(R, C_{a}\right) \\
& \stackrel{\mathrm{RHom}_{R}\left(\chi^{C}, C_{a}\right)^{-1}}{\longrightarrow} \mathrm{RHom}_{R}\left(\mathrm{RHom}_{R}(C, C), C_{a}\right) \\
& \simeq \quad \operatorname{RHom}_{R}\left(\mathrm{RHom}_{R}\left(C_{a} \oplus C_{1-a}, C\right), C_{a}\right) \\
& \simeq \mathrm{RHom}_{R}\left(\mathrm{RHom}_{R}\left(C_{a}, C\right), C_{a}\right) \text {. }
\end{aligned}
$$

Thus, for each idempotent $a$ there exists a canonical $C$-rigid pair $\left(C_{a}, \gamma_{a}\right)$.

Theorem 7.2. Let $C$ be a semidualizing complex.

A complex $M \in \mathrm{D}_{\mathrm{b}}^{\mathrm{f}}(R)$ is $C$-rigid if and only if it satisfies

$$
M \simeq C_{a} \quad \text { in } \mathrm{D}(R)
$$

for some idempotent a in $R$; such an idempotent is determined by the condition

$$
\operatorname{Supp}_{R} M=\{\mathfrak{p} \in \operatorname{Spec} R \mid \mathfrak{p} \not \supset a\} .
$$

Proof. The "if" part comes from Example 7.1, so assume that $M$ is $C$-rigid.

Set $L=\operatorname{RHom}_{R}(M, C)$ and let $M \simeq \operatorname{RHom}_{R}(L, M)$ be a rigidifying isomorphism. Theorem 4.9 produces a unique idempotent $a$ in $R$ satisfying (7.2.2), and such that the complex $L_{a}$ is invertible in $\mathrm{D}\left(R_{a}\right)$. Hence, $L_{a}$ is derived $C_{a}$-reflexive in $\mathrm{D}\left(R_{a}\right)$ by Lemma 5.4. Thus, $\mathrm{RHom}_{R_{a}}\left(M_{a}, C_{a}\right)$ is derived $C_{a}$-reflexive, and hence so is $M_{a}$, by Theorem 3.3. This explains the second isomorphism below:

$\operatorname{RHom}_{R_{a}}\left(L_{a}, C_{a}\right) \simeq \operatorname{RHom}_{R_{a}}\left(\operatorname{RHom}_{R_{a}}\left(M_{a}, C_{a}\right), C_{a}\right) \simeq M_{a} \simeq \operatorname{RHom}_{R_{a}}\left(L_{a}, M_{a}\right)$.

The third one is a localization of the rigidifying isomorphism. Hence $M_{a} \simeq C_{a}$ in $\mathrm{D}\left(R_{a}\right)$; see 5.2. It remains to note that one has $M \simeq M_{a}$ in $\mathrm{D}(R)$; see 4.8.

A morphism of $C$-rigid pairs is a commutative diagram

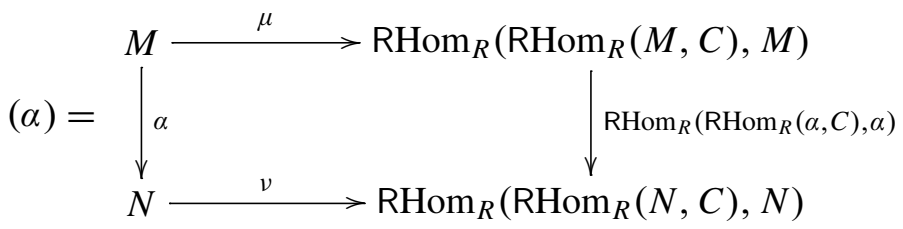

in $\mathrm{D}(R)$. The $C$-rigid pairs and their morphisms form a category, where composition is given by $(\beta)(\alpha)=(\beta \alpha)$ and $\mathrm{id}^{(M, \mu)}=\left(\mathrm{id}^{M}\right)$.

The next result explains the name 'rigid complex'. The result is deduced from Theorem 7.2 by transposing a beautiful observation of Yekutieli and Zhang [2008, proof of 4.4]: A morphism of rigid pairs is a natural isomorphism from a functor in $M$ that is linear to one that is quadratic, so it must be given by an idempotent. 
Theorem 7.3. If $C$ is a semidualizing complex and $(M, \mu)$ and $(N, v)$ are $C$-rigid pairs in $\mathrm{D}_{\mathrm{b}}^{\mathrm{f}}(R)$, then the following conditions are equivalent.

(i) There is an equality $\operatorname{Supp}_{R} N=\operatorname{Supp}_{R} M$.

(ii) There is an isomorphism $M \simeq N$ in $\mathrm{D}(R)$.

(iii) There is a unique isomorphism of $C$-rigid pairs $(M, \mu) \simeq(N, v)$.

Proof. (i) $\Longrightarrow$ (iii). Let $\alpha: C_{a} \stackrel{\simeq}{\rightarrow} M$ be an isomorphism in $\mathrm{D}(R)$ given by (7.2.1), with $a$ the idempotent defined by formula (7.2.2). It suffices to prove that $(M, \mu)$ is uniquely isomorphic to the $C$-rigid pair $\left(C_{a}, \gamma_{a}\right)$ from Example 7.1. Since it is equivalent to prove the same in $\mathrm{D}\left(R_{a}\right)$, we may replace $R$ by $R_{a}$ and drop all references to localization at $\{1, a\}$.

Set $\widetilde{\alpha}=\operatorname{RHom}_{R}\left(\operatorname{RHom}_{R}(\alpha, C), \alpha\right)$ : this is an isomorphism, and hence so is $\alpha^{-1} \circ \mu^{-1} \circ \tilde{\alpha} \circ \gamma: C \rightarrow C$. As $C$ is semidualizing, there is an isomorphism

$$
\mathrm{H}_{0}\left(\chi^{C}\right): R \stackrel{\cong}{\rightarrow} \mathrm{H}_{0}\left(\operatorname{RHom}_{R}(C, C)\right)=\operatorname{Hom}_{\mathrm{D}(R)}(C, C),
$$

of rings, so $\alpha^{-1} \circ \mu^{-1} \circ \widetilde{\alpha} \circ \gamma=\mathrm{H}_{0}\left(\chi^{C}\right)(u)$ for some unit $u$ in $R$. The next computation shows that $\left(u^{-1} \alpha\right):(C, \gamma) \rightarrow(M, \mu)$ is an isomorphism of $C$-rigid pairs:

$$
\begin{aligned}
\operatorname{RHom}_{R}\left(\operatorname{RHom}_{R}\left(u^{-1} \alpha, C\right), u^{-1} \alpha\right) \circ \gamma & =u^{-2}(\widetilde{\alpha} \circ \gamma) \\
& =u^{-2} \cdot u(\mu \circ \alpha) \\
& =\mu \circ\left(u^{-1} \alpha\right) .
\end{aligned}
$$

Let $(\beta):(C, \gamma) \rightarrow(M, \mu)$ also be such an isomorphism. The isomorphism $\mathrm{H}_{0}\left(\chi^{C}\right)$ implies that in $\mathrm{D}(R)$ one has $\beta^{-1} \circ u^{-1} \alpha=v$ id $^{C}$ for some unit $v \in R$, whence $v \mathrm{id}^{C}$ is a rigid endomorphism of the rigid pair $(C, \gamma)$. Thus

$$
\begin{aligned}
v \gamma & =\gamma \circ\left(v \mathrm{id}^{C}\right) \\
& =\operatorname{RHom}_{R}\left(\operatorname{RHom}_{R}\left(v \mathrm{id}^{C}, C\right), v \mathrm{id}^{C}\right) \circ \gamma \\
& =v^{2} \operatorname{RHom}_{R}\left(\operatorname{RHom}_{R}\left(\mathrm{id}^{C}, C\right), \mathrm{id}^{C}\right) \circ \gamma \\
& =v^{2} \gamma .
\end{aligned}
$$

As $v$ and $\gamma$ are invertible one gets $(v-1) \mathrm{id}^{C}=0$, hence $v-1 \in \operatorname{Ann}_{R} C=0$. This gives $v=1$, from where one obtains $\beta^{-1} \circ u^{-1} \alpha=\mathrm{id}^{C}$, and finally $(\beta)=\left(u^{-1} \alpha\right)$.

(iii) $\Longrightarrow$ (ii) $\Longrightarrow$ (i). These implications are evident.

An alternative formulation of the preceding result is sometimes useful.

Remark 7.4. Let $(M, \mu)$ be a $C$-rigid pair in $\mathrm{D}_{\mathrm{b}}^{\mathrm{f}}(R)$, and $N$ a complex in $\mathrm{D}_{\mathrm{b}}^{\mathrm{f}}(R)$. 
For each isomorphism $\alpha: N \stackrel{\simeq}{\rightarrow} M$ in $\mathrm{D}(R)$, set

$$
\rho(\alpha)=\left(\operatorname{RHom}_{R}\left(\operatorname{RHom}_{R}(\alpha, C), \alpha\right)\right)^{-1} \circ \mu \circ \alpha ;
$$

this is a morphism from $N$ to $\operatorname{RHom}_{R}\left(\operatorname{RHom}_{R}(N, C), N\right)$.

Theorem 7.3 shows that the assignment $\alpha \mapsto(N, \rho(\alpha))$ yields a bijection

\{isomorphisms from $N$ to $M\} \leftrightarrow$ rigid pairs $(N, v)$ isomorphic to $(M, \mu)$ \}

We finish with a converse, of sorts, to Example 7.1.

Proposition 7.5. If $C$ in $\mathrm{D}_{\mathrm{b}}^{\mathrm{f}}(R)$ is $C$-rigid, then there exist an idempotent a in $R$, a semidualizing complex $B$ for $R_{a}$, and an isomorphism $C \simeq B$ in $\mathrm{D}(R)$.

Proof. By hypothesis, $C \simeq \mathrm{RHom}_{R}\left(\mathrm{RHom}_{R}(C, C), C\right)$. Theorem 4.9 and 4.8 provide an idempotent $a \in R$ such that the $R_{a}$-module $\mathrm{H}_{0}\left(\mathrm{RHom}_{R}(C, C)_{a}\right)$ is invertible and in $\mathrm{D}(R)$ there are natural isomorphisms $C \simeq C_{a}$ and

$$
\mathrm{H}_{0}\left(\mathrm{RHom}_{R}(C, C)_{a}\right) \simeq \mathrm{RHom}_{R}(C, C)_{a} \simeq \mathrm{RHom}_{R_{a}}\left(C_{a}, C_{a}\right) .
$$

It follows that the homothety map

$$
\chi: R_{a} \rightarrow \operatorname{Hom}_{\mathrm{D}\left(R_{a}\right)}\left(C_{a}, C_{a}\right) \cong \mathrm{H}_{0}\left(\mathrm{RHom}_{R_{a}}\left(C_{a}, C_{a}\right)\right)
$$

turns $\operatorname{Hom}_{\mathrm{D}\left(R_{a}\right)}\left(C_{a}, C_{a}\right)$ into both an invertible $R_{a}$-module and an $R_{a}$-algebra. Localizing at prime ideals of $R_{a}$, one sees that such a $\chi$ must be an isomorphism; so the proposition holds with $B=C_{a}$.

\section{Relative dualizing complexes}

In this section $K$ denotes a commutative noetherian ring, $S$ a commutative ring, and $\sigma: K \rightarrow S$ a homomorphism of rings that is assumed to be essentially of finite type: This means that $\sigma$ can be factored as a composition

$$
K \hookrightarrow K\left[x_{1}, \ldots, x_{e}\right] \rightarrow W^{-1} K\left[x_{1}, \ldots, x_{e}\right]=Q \rightarrow S
$$

of homomorphisms of rings, where $x_{1}, \ldots, x_{e}$ are indeterminates, $W$ is a multiplicatively closed set, the first two maps are canonical, the equality defines $Q$, and the last arrow is surjective; the map $\sigma$ is of finite type if one can choose $W=\{1\}$.

As usual, $\Omega_{Q \mid K}$ stands for the $Q$-module of Kähler differentials; for each $n \in \mathbb{Z}$ we set $\Omega_{Q \mid K}^{n}=\bigwedge_{Q}^{n} \Omega_{Q \mid K}$. Fixing the factorization (8.0.1), we define a relative dualizing complex for $\sigma$ by means of the following equality:

$$
D^{\sigma}=\Sigma^{e} \operatorname{RHom}_{Q}\left(S, \Omega_{Q \mid K}^{e}\right) .
$$

Our goal here is to determine when $D^{\sigma}$ is semidualizing, invertible, or dualizing. It turns out that each one of these properties is equivalent to some property of the 
homomorphism $\sigma$, which has been studied earlier in a different context. We start by introducing notation and terminology that will be used throughout the section.

For every $\mathfrak{q}$ in Spec $S$ we let $\mathfrak{q} \cap K$ denote the prime ideal $\sigma^{-1}(\mathfrak{q})$ of $K$, and write $\sigma_{\mathfrak{q}}: K_{\mathfrak{q} \cap K} \rightarrow S_{\mathfrak{q}}$ for the induced local homomorphism; it is essentially of finite type.

Recall that a ring homomorphism $\dot{\sigma}: K \rightarrow P$ is said to be (essentially) smooth if it is (essentially) of finite type, flat, and for each ring homomorphism $K \rightarrow k$, where $k$ is a field, the ring $k \otimes_{K} P$ is regular; by [Grothendieck 1967, 17.5.1] this notion of smoothness is equivalent to the one defined in terms of lifting of homomorphisms. When $\dot{\sigma}$ is essentially smooth $\Omega_{P \mid K}$ is finite projective over $P$; in case $\Omega_{P \mid K}$ has rank $d$ (see 4.7) we say that $\dot{\sigma}$ has relative dimension $d$. The $P$-module $\Omega_{P \mid K}^{d}$ is then invertible.

An (essential) smoothing of $\sigma$ (of relative dimension $d$ ) is a decomposition

$$
K \stackrel{\dot{\sigma}}{\rightarrow} P \stackrel{\sigma^{\prime}}{\rightarrow} S
$$

of $\sigma$ with $\dot{\sigma}$ (essentially) smooth of fixed relative dimension (equal to $d$ ) and $\sigma^{\prime}$ finite, meaning that $S$ is a finite $P$-module via $\sigma^{\prime}$; an essential smoothing of $\sigma$ always exists; see (8.0.1).

8.1. Basic properties. Fix an essential smoothing (8.0.3) of relative dimension $d$.

8.1.1. By [Avramov et al. 2010a, 1.1], there exists an isomorphism

$$
D^{\sigma} \simeq \Sigma^{d} \operatorname{RHom}_{P}\left(S, \Omega_{P \mid K}^{d}\right) \quad \text { in } \mathrm{D}(S) .
$$

8.1.2. For each $M$ in $\mathrm{D}_{\mathrm{b}}^{\mathrm{f}}(S)$ there are isomorphisms

$$
\begin{aligned}
\operatorname{RHom}_{S}\left(M, D^{\sigma}\right) & =\operatorname{RHom}_{S}\left(M, \Sigma^{d} \operatorname{RHom}_{P}\left(S, \Omega_{P \mid K}^{d}\right)\right) \\
& \simeq \Sigma^{d} \operatorname{RHom}_{P}\left(M, \Omega_{P \mid K}^{d}\right) \\
& \simeq \operatorname{RHom}_{P}(M, P) \otimes_{P} \Sigma^{d} \Omega_{P \mid K}^{d}
\end{aligned}
$$

in $\mathrm{D}(S)$, because $\Omega_{P \mid K}^{d}$ is an invertible $P$-module.

Proposition 8.1.3. If $U \subseteq K$ and $V \subseteq S$ are multiplicatively closed sets satisfying $\sigma(U) \subseteq V$, and $\widetilde{\sigma}: U^{-1} K \rightarrow V^{-1} S$ is the induced map, then one has

$$
d^{\widetilde{\sigma}} \simeq v^{-1} d^{\sigma} \quad \text { in } \mathrm{D}\left(v^{-1} s\right) .
$$

Proof. set $v^{\prime}=\sigma^{\prime-1}(v)$. in the induced factorization $u^{-1} k \rightarrow\left(v^{\prime}\right)^{-1} p \rightarrow v^{-1} s$ of $\tilde{\sigma}$, the first map is essentially smooth of relative dimension $d$ and the second one is finite. the first and the last isomorphisms in the next chain hold by 8.1.1, the rest 
because localization commutes with modules of differentials and exterior powers:

$$
\begin{aligned}
D^{\widetilde{\sigma}} & \simeq \Sigma^{d} \operatorname{RHom}_{\left(V^{\prime}\right)^{-1} P}\left(\left(V^{\prime}\right)^{-1} S, \Omega_{\left(V^{\prime}\right)^{-1} P \mid U^{-1} K}^{d}\right) \\
& \simeq \Sigma^{d} \operatorname{RHom}_{\left(V^{\prime}\right)^{-1} P}\left(\left(V^{\prime}\right)^{-1} S,\left(V^{\prime}\right)^{-1} \Omega_{P \mid K}^{d}\right) \\
& \simeq\left(V^{\prime}\right)^{-1} \Sigma^{d} \operatorname{RHom}_{P}\left(S, \Omega_{P \mid K}^{d}\right) \\
& \simeq V^{-1} D^{\sigma} .
\end{aligned}
$$

Proposition 8.1.4. If $\varphi: S \rightarrow T$ is a finite homomorphism of rings, then for the map $\tau=\varphi \sigma: K \rightarrow T$ there is an isomorphism

$$
D^{\tau} \simeq \operatorname{RHom}_{S}\left(T, D^{\sigma}\right) \quad \text { in } \mathrm{D}(T) .
$$

Proof. The result comes from the following chain of isomorphisms:

$$
\begin{aligned}
D^{\tau} & \simeq \Sigma^{d} \operatorname{RHom}_{P}\left(T, \Omega_{P \mid K}^{d}\right) \\
& \simeq \operatorname{RHom}_{S}\left(T, \Sigma^{d} \operatorname{RHom}_{P}\left(S, \Omega_{P \mid K}^{d}\right)\right) \\
& =\operatorname{RHom}_{S}\left(T, D^{\sigma}\right),
\end{aligned}
$$

where the first one is obtained from the factorization $K \stackrel{\kappa}{\rightarrow} P \stackrel{\varphi \sigma^{\prime}}{\rightarrow} T$ of $\tau$ and the second one by adjunction.

8.2. Derived $D^{\sigma}$-reflexivity. A standard calculation shows that derived $D^{\sigma}$-reflexivity can be read off any essential smoothing (see (8.0.3)).

Proposition 8.2.1. A complex $M$ in $\mathrm{D}(S)$ is derived $D^{\sigma}$-reflexive if and only if $M$ is derived $P$-reflexive when viewed as a complex in $\mathrm{D}(P)$.

Proof. Evidently, $M$ is in $\mathrm{D}_{\mathrm{b}}^{\mathrm{f}}(S)$ if and only if it is in $\mathrm{D}_{\mathrm{b}}^{\mathrm{f}}(P)$. From 8.1.2 one sees that $\operatorname{RHom}_{S}\left(M, D^{\sigma}\right)$ is in $\mathrm{D}_{\mathrm{b}}^{\mathrm{f}}(S)$ if and only if $\operatorname{RHom}_{P}(M, P)$ is in $\mathrm{D}_{\mathrm{b}}^{\mathrm{f}}(P)$.

Set $\Omega=\Sigma^{d} \Omega_{P \mid K}^{d}$, where $d$ is the relative dimension of $K \rightarrow P$, and let $\Omega \rightarrow I$ be a semiinjective resolution in $\mathrm{D}(P)$. Thus, $D^{\sigma}$ is isomorphic to $\operatorname{Hom}_{P}(S, I)$ in $\mathrm{D}(S)$. The biduality morphism $\delta_{M}^{\Omega}$ in $\mathrm{D}(P)$ is realized by a morphism

$$
M \rightarrow \operatorname{Hom}_{P}\left(\operatorname{Hom}_{P}(M, I), I\right)
$$

of complexes of $S$-modules; see (2.0.1). Its composition with the natural isomorphism of complexes of $S$-modules

$$
\operatorname{Hom}_{P}\left(\operatorname{Hom}_{P}(M, I), I\right) \cong \operatorname{Hom}_{S}\left(\operatorname{Hom}_{S}\left(M, \operatorname{Hom}_{P}(S, I)\right), \operatorname{Hom}_{P}(S, I)\right)
$$

represents the morphism $\delta_{M}^{D^{\sigma}}$ in $\mathrm{D}(S)$. It follows that $M$ is derived $D^{\sigma}$-reflexive if and only if it is derived $\Omega$-reflexive. Since $\Omega$ is an invertible $P$-module, the last condition is equivalent — by Lemma 5.4 - to the derived $P$-reflexivity of $M$. 
A complex $M$ in $\mathrm{D}_{+}(S)$ is said to have finite flat dimension over $K$ if $M$ is isomorphic in $\mathrm{D}(K)$ to a bounded complex of flat $K$-modules; we then write $\mathrm{fd}_{K} M<\infty$.

When $\mathrm{fd}_{K} S$ is finite we say that $\sigma$ is of finite flat dimension and write $\mathrm{fd} \sigma<\infty$.

8.2.2. A complex $M$ in $\mathrm{D}_{\mathrm{b}}^{\mathrm{f}}(S)$ satisfies $\mathrm{fd}_{K} M<\infty$ if and only if it is perfect in $\mathrm{D}(P)$ for some (equivalently, any) factorization (8.0.3) of $\sigma$; see [Avramov et al. 2010a, beginning of $\S 6]$.

Corollary 8.2.3. A complex $M$ in $\mathrm{D}_{\mathrm{b}}^{\mathrm{f}}(S)$ with $\mathrm{fd}_{K} M<\infty$ is derived $D^{\sigma}$-reflexive. Proof. By 8.2.2 the complex $M$ is perfect in $\mathrm{D}(P)$. It is then obviously derived $P$-reflexive, and so is derived $D^{\sigma}$-reflexive by the previous proposition.

8.3. Gorenstein base rings. Relative dualizing complexes and their absolute counterparts (see 6.2) are compared in the next result, where the "if" part is classical.

Theorem 8.3.1. The complex $D^{\sigma}$ is strongly pointwise dualizing for $S$ if and only if the ring $K_{\mathfrak{q} \cap K}$ is Gorenstein for every prime ideal $\mathfrak{q}$ of $S$.

Proof. Factor $\sigma$ as in (8.0.1) and set $\mathfrak{p}=\mathfrak{q} \cap K$. The homomorphism $\sigma_{\mathfrak{q}}: K_{\mathfrak{p}} \rightarrow S_{\mathfrak{q}}$ satisfies $\left(D^{\sigma}\right)_{\mathfrak{q}} \cong D^{\sigma_{\mathfrak{q}}}$ by Proposition 8.1.3. Localizing, we may assume that $\sigma$ is a local homomorphism $(K, \mathfrak{p}) \rightarrow(S, \mathfrak{q})$, and that the ring $Q$ is local. As the ring $Q / \mathfrak{p} Q$ is regular, $K$ is Gorenstein if and only if $Q$ is too; see [Matsumura 1986, 23.4]. Thus, replacing $Q$ with $K$ we may further assume that $\sigma$ is surjective.

If $K$ is Gorenstein, then $D^{\sigma}=\operatorname{RHom}_{K}(S, K)$ holds so it is dualizing for $S$ by Corollaries 6.2.3 and 6.2.4.

When $D^{\sigma}$ is dualizing for $S$, the residue field $k=S / \mathfrak{q}$ is derived $D^{\sigma}$-reflexive; see Theorem 6.2.2. By Proposition 8.2.1 it is also derived $K$-reflexive, which implies $\operatorname{Ext}_{K}^{n}(k, K)=0$ for $n \gg 0$. Thus, $K$ is Gorenstein; see [Matsumura 1986, 18.1].

8.4. Homomorphisms offinite G-dimension. When the $P$-module $S$ has finite $\mathrm{G}$ dimension (see 6.3) we say that $\sigma$ has finite $G$-dimension and write G-dim $\sigma<\infty$. By the following result, this notion is independent of the choice of factorization.

Proposition 8.4.1. The following conditions are equivalent.

(i) $D^{\sigma}$ is semidualizing for $S$.

(ii) $\sigma$ has finite G-dimension.

(iii) $\sigma_{\mathfrak{n}}$ has finite $G$-dimension for each $\mathfrak{n} \in \operatorname{Max} S$.

Proof. (i) $\Longleftrightarrow$ (ii). By Proposition 3.1, $D^{\sigma}$ is semidualizing for $S$ if and only if $S$ is derived $D^{\sigma}$-reflexive. By Proposition 8.2.1 this is equivalent to $S$ being derived $P$-reflexive in $\mathrm{D}(P)$, and hence, by 6.3.1, to $S$ having finite G-dimension over $P$.

(ii) $\Longleftrightarrow$ (iii). Proposition 8.1.3 yields an isomorphism $D^{\sigma_{\mathfrak{n}}} \simeq\left(D^{\sigma}\right)_{\mathfrak{n}}$ for each $\mathfrak{n}$. Given (i) $\Longleftrightarrow$ (ii), the desired equivalence follows from Proposition 3.1. 
Combining the proposition with Theorem 8.3.1 and Corollary 8.2.3, one obtains: Corollary 8.4.2. Each condition below implies that $\sigma$ has finite $G$-dimension:

(i) The ring $K_{\mathfrak{n} \cap K}$ is Gorenstein for every $\mathfrak{n} \in \operatorname{Max} S$.

(ii) The homomorphism $\sigma$ has finite flat dimension.

Notes 8.4.3. A notion of finite G-dimension that applies to arbitrary local homomorphisms is defined in [Avramov and Foxby 1997]. Proposition 8.4.1 and [Avramov and Foxby 1997, 4.3, 4.5] show that the definitions agree when both apply; thus, Corollary 8.4.2 recovers [Avramov and Foxby 1997, 4.4.1, 4.4.2].

8.5. Relative rigidity. Proposition 8.4 .1 and Theorem 7.2 yield:

Theorem 8.5.1. Assume that $\sigma$ has finite G-dimension.

A complex $M$ in $\mathrm{D}_{\mathrm{b}}^{\mathrm{f}}(S)$ is $D^{\sigma}$-rigid if and only if it is isomorphic to $D_{a}^{\sigma}$ for some idempotent $a \in S$; such an idempotent is uniquely defined.

This theorem greatly strengthens some results of [Yekutieli and Zhang 2009], where rigidity is defined using a derived version of Hochschild cohomology, due to Quillen: there is a functor

$$
\mathrm{RHom}_{S \otimes_{K}^{\mathrm{L}} S}\left(S,-\otimes_{K}^{\mathrm{L}}-\right): \mathrm{D}(S) \times \mathrm{D}(S) \rightarrow \mathrm{D}(S)
$$

(see [Avramov et al. 2010a, §3] for details of the construction) which has the following properties:

8.5.2. Quillen's derived Hochschild cohomology modules (see [Quillen 1970, §3]) are given by

$$
\operatorname{Ext}_{S \otimes_{K}^{\llcorner} S}^{n}\left(S, M \otimes_{K}^{\mathrm{L}} N\right)=\mathrm{H}_{-n}\left(\operatorname{RHom}_{S \otimes_{K}^{\llcorner} S}\left(S, M \otimes_{K}^{\mathrm{L}} N\right)\right) .
$$

8.5.3. When $S$ is $K$-flat one can replace $S \otimes_{K}^{\mathrm{L}} S$ with $S \otimes_{K} S$; see [Avramov et al. 2010a, Remark 3.4].

8.5.4. When $\mathrm{fd} \sigma$ is finite, for every complex $M$ in $\mathrm{D}_{\mathrm{b}}^{\mathrm{f}}(S)$ with $\mathrm{fd}_{K} M<\infty$ and for every complex $N$ in $\mathrm{D}(S)$, by [Avramov et al. 2010a, Theorem 4.1] there exists an isomorphism

$$
\mathrm{RHom}_{S \otimes_{K}^{\mathrm{L}} S}\left(S, M \otimes_{K}^{\mathrm{L}} N\right) \simeq \mathrm{RHom}_{S}\left(\operatorname{RHom}_{S}\left(M, D^{\sigma}\right), N\right) \quad \text { in } \mathrm{D}(S) .
$$

Yekutieli and Zhang [2008, 4.1] define $M$ in $\mathrm{D}(S)$ to be rigid relative to $K$ if $M$ is in $\mathrm{D}_{\mathrm{b}}^{\mathrm{f}}(S)$, satisfies $\mathrm{fd}_{K} M<\infty$, and admits a rigidifying isomorphism

$$
\mu: M \stackrel{\simeq}{\rightarrow} \operatorname{RHom}_{S \otimes_{K}^{\llcorner} S}\left(S, M \otimes_{K}^{\mathrm{L}} M\right) \quad \text { in } \mathrm{D}(S) .
$$

By 8.5.3, when $K$ is a field, this coincides with the notion introduced by Van den Bergh [1997, 8.1]. On the other hand, (7.0.1) and 8.5.4, applied with $N=M$, give: 
8.5.5. When $\mathrm{fd} \sigma$ is finite, $M$ in $\mathrm{D}_{\mathrm{b}}^{\mathrm{f}}(S)$ is rigid relative to $K$ if and only if $\mathrm{fd}_{K} M$ is finite and $M$ is $D^{\sigma}$-rigid.

From Theorems 8.5.1 and 8.3.1 we now obtain:

Theorem 8.5.6. Assume that $K$ is Gorenstein and $\mathrm{fd} \sigma$ is finite.

The complex $D^{\sigma}$ then is pointwise dualizing for $S$ and is rigid relative to $K$.

A complex $M$ in $\mathrm{D}_{\mathrm{b}}^{\mathrm{f}}(S)$ is rigid relative to $K$ if and only if $D_{a}^{\sigma} \cong M$ holds for some idempotent a in $S$. More precisely, when $\delta$ and $\mu$ are rigidifying isomorphisms for $D^{\sigma}$ and $M$, respectively, there exists a commutative diagram

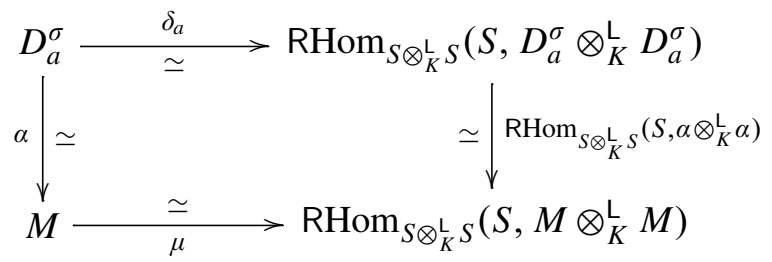

where both the idempotent $a$ and the isomorphism $\alpha$ are uniquely defined.

In [Yekutieli and Zhang 2009] the ring $K$ is assumed regular of finite Krull dimension. This implies $\mathrm{fd}_{K} M<\infty$ for all $M \in \mathrm{D}_{\mathrm{b}}^{\mathrm{f}}(S)$, so fd $\sigma<\infty$ holds, and also that $S$ is of finite Krull dimension, since it is essentially of finite type over $K$. Therefore [Yekutieli and Zhang 2009, 1.1(a), alias 3.6(a) and 1.2, alias 3.10] are special cases of Theorem 8.5.6.

There also is a converse, stemming from 6.2.1 and Theorem 8.3.1.

Finally, we address a series of comments made at the end of [Yekutieli and Zhang 2009, §3]; they are given in quotation marks, but notation and references are changed to match ours.

Notes 8.5.7. The paragraph preceding [Yekutieli and Zhang 2009, 3.10] reads: "Next comes a surprising result that basically says 'all rigid complexes are dualizing'. The significance of this result is yet unknown." It states: If $K$ and $S$ are regular, $\operatorname{dim} S$ is finite, and $S$ has no idempotents other that 0 and 1, then a rigid complex is either zero or dualizing.

Theorem 7.2 provides an explanation of this phenomenon: Under these conditions $S$ has finite global dimension, so every semidualizing complex is dualizing.

Notes 8.5.8. Concerning [Yekutieli and Zhang 2009, 3.14]: "The standing assumptions that the base ring $K$ has finite global dimension seems superfluous." See Theorem 8.5.6.

"However, it seems necessary for $K$ to be Gorenstein - see [Yekutieli and Zhang 2009, Example 3.16]." Compare Theorems 8.5.1 and 8.5.6.

"A similar reservation applies to the assumption that $S$ is regular in Theorem 3.10 (Note the mistake in [Yekutieli and Zhang 2008, Theorem 0.6]: there too $S$ 
has to be regular)." Theorem 8.5.6 shows that the regularity hypothesis can be weakened significantly.

8.6. Quasi-Gorenstein homomorphisms. The map $\sigma$ is said to be quasi-Gorenstein if in (8.0.1) for each $\mathfrak{n} \in \operatorname{Max} S$ the $Q_{\mathfrak{n} \cap Q}$-module $S_{\mathfrak{n}}$ has finite G-dimension and satisfies $\operatorname{RHom}_{Q_{\mathfrak{n} \cap Q}}\left(S_{\mathfrak{n}}, Q_{\mathfrak{n} \cap S}\right) \simeq \Sigma^{r(\mathfrak{n})} S_{\mathfrak{n}}$ for some $r(\mathfrak{n}) \in \mathbb{Z}$; see [Avramov and Foxby 1997, 5.4, 6.7, 7.8, 8.4]; when this holds $\sigma$ has finite $G$-dimension by Corollary 6.3.4.

By part (i) of the next theorem, quasi-Gorensteinness is a property of $\sigma$, not of the factorization. The equivalence of (ii) and (iii) also follows from [Avramov and Iyengar 2008, 2.2].

Theorem 8.6.1. The following conditions are equivalent:

(i) $D^{\sigma}$ is invertible in $\mathrm{D}(S)$.

(i') $D^{\sigma}$ is derived $S$-reflexive in $\mathrm{D}(S)$ and $\mathrm{G}-\operatorname{dim} \sigma<\infty$.

(ii) $\sigma$ is quasi-Gorenstein.

(iii) $\operatorname{Ext}_{P}(S, P)$ is an invertible graded $S$-module.

Proof. (i) $\Longleftrightarrow\left(\mathrm{i}^{\prime}\right)$. This results from Proposition 8.4.1 and Corollary 5.7.

(i) $\Longleftrightarrow$ (iii). By 8.1.2, one has $D^{\sigma} \simeq \Sigma^{d} \operatorname{RHom}_{P}(S, P) \otimes_{P}^{\mathrm{L}} \Omega_{P \mid K}^{d}$ in $\mathrm{D}(S)$. It implies that $D^{\sigma}$ is invertible in $\mathrm{D}(S)$ if and only if $\operatorname{RHom}_{P}(S, P)$ is. By Proposition 5.1 , the latter condition holds if and only if $\operatorname{Ext}_{P}(S, P)$ is invertible.

(i') \& (iii) $\Longrightarrow$ (ii). Indeed, for every $\mathfrak{n} \in \operatorname{Spec} S$ the finiteness of G-dim $\sigma$ implies that of G-dim $P_{P_{\mathfrak{n} \cap P}} S_{\mathfrak{n}}$, and the invertibility of $\operatorname{Ext}_{P}(S, P)$ implies an isomorphism $\mathrm{RHom}_{P_{\mathfrak{n} \cap P}}\left(S_{\mathfrak{n}}, P_{\mathfrak{n} \cap P}\right) \simeq \Sigma^{r(\mathfrak{n})} S_{\mathfrak{n}}$ for some $r(\mathfrak{n}) \in \mathbb{Z}$; see Proposition 5.1.

(ii) $\Longrightarrow$ (iii). This follows from Proposition 5.1 .

A quasi-Gorenstein homomorphism $\sigma$ with $\operatorname{fd}_{K} S<\infty$ is said to be Gorenstein; see [Avramov and Foxby 1997, 8.1]. When $\sigma$ is flat, it is Gorenstein if and only if for every $\mathfrak{q} \in \operatorname{Spec} S$ and $\mathfrak{p}=\mathfrak{q} \cap K$ the $\operatorname{ring}\left(K_{\mathfrak{p}} / \mathfrak{p} K_{\mathfrak{p}}\right) \otimes_{K} S$ is Gorenstein; see [Avramov and Foxby 1997, 8.3]. The next result uses derived Hochschild cohomology; see 8.5.2. For flat $\sigma$ it is proved in [Avramov and Iyengar 2008, 2.4].

Theorem 8.6.2. The map $\sigma$ is Gorenstein if and only if $\mathrm{fd} \sigma$ is finite and the graded $S$-module $\operatorname{Ext}_{S \otimes_{K}^{\mathrm{L}} S}\left(S, S \otimes_{K}^{\mathrm{L}} S\right)$ is invertible. When $\sigma$ is Gorenstein one has

$$
D^{\sigma} \simeq \operatorname{Ext}_{S \otimes_{K}^{\mathrm{L}} S}\left(S, S \otimes_{K}^{\mathrm{L}} S\right)^{-1} \quad \text { in } \mathrm{D}(S)
$$

and one can replace $S \otimes_{K}^{\mathrm{L}} S$ with $S \otimes_{K} S$ in case $\sigma$ is flat. 
Proof. We may assume that $\mathrm{fd} \sigma$ is finite. One then gets an isomorphism

$$
\operatorname{RHom}_{S}\left(D^{\sigma}, S\right) \simeq \operatorname{RHom}_{S \otimes_{K}^{\llcorner} S}\left(S, S \otimes_{K}^{\llcorner} S\right) \quad \text { in } \quad \mathrm{D}(S)
$$

from 8.5.4 with $M=S=N$. The following equivalences then hold:

$\sigma$ is Gorenstein $\Longleftrightarrow D^{\sigma}$ is invertible

$$
\begin{aligned}
& \Longleftrightarrow \operatorname{RHom}_{S}\left(D^{\sigma}, S\right) \text { is invertible } \\
& \Longleftrightarrow \operatorname{RHom}_{S \otimes_{K}^{\llcorner} S}\left(S, S \otimes_{K}^{\mathrm{L}} S\right) \text { is invertible } \\
& \Longleftrightarrow \operatorname{Ext}_{S \otimes_{K}^{\llcorner} S}\left(S, S \otimes_{K}^{\mathrm{L}} S\right) \text { is invertible }
\end{aligned}
$$

[by Theorem 8.6.1]

[by Proposition 5.1]

[by (8.0.4)]

[by Proposition 5.1]

When $D^{\sigma}$ is invertible, (8.0.4) and 4.6 yield isomorphisms

$$
\left(D^{\sigma}\right)^{-1} \simeq \operatorname{RHom}_{S \otimes_{K}^{\llcorner} S}\left(S, S \otimes_{K}^{\mathrm{L}} S\right) \simeq \operatorname{Ext}_{S \otimes_{K}^{\llcorner} S}\left(S, S \otimes_{K}^{\mathrm{L}} S\right) \quad \text { in } \mathrm{D}(S),
$$

whence the desired expression for $D^{\sigma}$. The last assertion comes from 8.5.3.

Combining Theorem 8.6.2, Proposition 8.1.4, and the isomorphism in 8.1.2, we see that $D^{\sigma}$ can be computed from factorizations through arbitrary Gorenstein homomorphisms — not just through essentially smooth ones, as provided by 8.1.1.

Corollary 8.6.3. If $K \stackrel{\varkappa}{\rightarrow} Q \stackrel{\varkappa^{\prime}}{\rightarrow} S$ is a factorization of $\sigma$ with $\varkappa$ Gorenstein and $\varkappa^{\prime}$ finite, then there is an isomorphism

$$
D^{\sigma} \simeq \operatorname{RHom}_{Q}(S, Q) \otimes_{Q} \operatorname{Ext}_{Q \otimes_{K}^{L} Q}\left(Q, Q \otimes_{K}^{\mathrm{L}} Q\right)^{-1} \quad \text { in } \mathrm{D}(S) .
$$

\section{Appendix: Homological invariants}

Let $R$ be a commutative noetherian ring.

Complexes of $R$-modules have differentials of degree -1 . Modules are identified with complexes concentrated in degree zero. For every graded $R$-module $H$ we set

$$
\inf H=\inf \left\{n \in \mathbb{Z} \mid H_{n} \neq 0\right\} \quad \text { and } \quad \sup H=\sup \left\{n \in \mathbb{Z} \mid H_{n} \neq 0\right\} .
$$

The amplitude of $H$ is the number amp $H=\sup H-\inf H$. Thus $H=0$ is equivalent to inf $H=\infty$; to $\sup H=-\infty$; to amp $H=-\infty$, and also to amp $H<0$.

We write $\mathrm{D}(R)$ for the derived category of $R$-modules, and $\Sigma$ for its translation functor. Various full subcategories of $\mathrm{D}(R)$ are used in this text. Our notation for them is mostly standard: the objects of $\mathrm{D}_{+}(R)$ are the complexes $M$ with $\inf \mathrm{H}(M)>-\infty$, those of $\mathrm{D}_{-}(R)$ are the complexes $M$ with $\sup \mathrm{H}(M)<\infty$, and $\mathrm{D}_{\mathrm{b}}(R)=\mathrm{D}_{+}(R) \cap \mathrm{D}_{-}(R)$. Also, $\mathrm{D}^{f}(R)$ is the category of complexes $M$ with $\mathrm{H}_{n}(M)$ finite for each $n \in \mathbb{Z}$, and we set $\mathrm{D}_{+}^{\mathrm{f}}(R)=\mathrm{D}^{\mathrm{f}}(R) \cap \mathrm{D}_{+}(R)$, etc. 
For complexes $M$ and $N$ in $\mathrm{D}(R)$ we write $M \otimes_{R}^{\mathrm{L}} N$ for the derived tensor product, $\operatorname{RHom}_{R}(M, N)$ for the derived complex of homomorphisms, and set

$$
\operatorname{Tor}_{n}^{R}(M, N)=\mathrm{H}_{n}\left(M \otimes_{R}^{\mathrm{L}} N\right) \quad \text { and } \operatorname{Ext}_{R}^{n}(M, N)=\mathrm{H}_{-n}\left(\operatorname{RHom}_{R}(M, N)\right) .
$$

Standard spectral sequence arguments give the following well known assertions:

A.1. For all complexes $M$ and $N$ in $\mathrm{D}(R)$ there are inequalities

$$
\begin{gathered}
\sup \mathrm{H}\left(\operatorname{RHom}_{R}(M, N)\right) \leq \sup \mathrm{H}(N)-\inf \mathrm{H}(M), \\
\inf \mathrm{H}\left(M \otimes_{R}^{\mathrm{L}} N\right) \geq \inf \mathrm{H}(M)+\inf \mathrm{H}(N) .
\end{gathered}
$$

If $M$ is in $\mathrm{D}_{+}^{\mathrm{f}}(R)$ and $N$ is in $\mathrm{D}_{-}^{\mathrm{f}}(R)$, then $\mathrm{RHom}_{R}(M, N)$ is in $\mathrm{D}_{-}^{\mathrm{f}}(R)$. If $M$ and $N$ are in $\mathrm{D}_{+}^{\mathrm{f}}(R)$, then so is $M \otimes_{R}^{\mathrm{L}} N$.

For ease of reference, we list some canonical isomorphisms:

A.2. Let $\mathfrak{m}$ be a maximal ideal of $R$ and set $k=R / \mathfrak{m}$. For all complexes $M$ in $\mathrm{D}(R)$ and $N$ in $\mathrm{D}_{-}^{\mathrm{f}}(R)$ there are isomorphisms of graded $k$-vector spaces

$$
\begin{gathered}
k \otimes_{R}^{\mathrm{L}} M \cong\left(k \otimes_{R}^{\mathrm{L}} M\right)_{\mathfrak{m}} \cong k \otimes_{R}^{\mathrm{L}} M_{\mathfrak{m}} \cong k \otimes_{R_{\mathfrak{m}}}^{\mathrm{L}} M_{\mathfrak{m}} ; \\
\operatorname{RHom}_{R}(k, N) \cong \operatorname{RHom}_{R}(k, N)_{\mathfrak{m}} \cong \operatorname{RHom}_{R}\left(k, N_{\mathfrak{m}}\right) \cong \operatorname{RHom}_{R_{\mathfrak{m}}}\left(k, N_{\mathfrak{m}}\right) .
\end{gathered}
$$

We write that $(R, \mathfrak{m}, k)$ is a local ring to indicate that $R$ is a commutative noetherian ring with unique maximal ideal $\mathfrak{m}$ and with residue field $k=R / \mathfrak{m}$.

The statements below may be viewed as partial converses to those in A.1.

A.3. Let $(R, \mathfrak{m}, k)$ be a local ring and $M$ a complex in $\mathrm{D}^{\mathrm{f}}(R)$.

If $\operatorname{RHom}_{R}(k, M)$ is in $\mathrm{D}_{-}(R)$, then $M$ is in $\mathrm{D}_{-}(R)$.

If $k \otimes_{R}^{\mathrm{L}} M$ is in $\mathrm{D}_{+}(R)$, then $M$ is in $\mathrm{D}_{+}(R)$.

See [Foxby and Iyengar 2003, 2.5, 4.5] for the original proofs. The proof of [Avramov and Iyengar 2008, 1.5] gives a shorter, simpler, argument for the second assertion; it can be adapted to cover the first one.

Many arguments in this paper utilize invariants of local rings with values in the ring $\mathbb{Z} \llbracket t \rrbracket\left[t^{-1}\right]$ of formal Laurent series in $t$ with integer coefficients. The order of such a series $F(t)=\sum_{n \in \mathbb{Z}} a_{n} t^{n}$ is the number

$$
\operatorname{ord}(F(t))=\inf \left\{n \in \mathbb{Z} \mid a_{n} \neq 0\right\} .
$$

To obtain the expressions for Poincaré series and Bass series in Lemmas A.4.3 and A.5.3 below, we combine ideas from Foxby's proofs [1977, 4.1, 4.2] with the results in A.3; this allows us to relax some boundedness conditions in [Foxby 1977]. 
A.4 (Poincaré series). For a local ring $(R, \mathfrak{m}, k)$ and for $M$ in $D_{+}^{f}(R)$, in view of A.1 the formula below defines a formal Laurent series, called the Poincaré series of $M$ :

$$
P_{M}^{R}(t)=\sum_{n \in \mathbb{Z}} \operatorname{rank}_{k} \operatorname{Tor}_{n}^{R}(k, M) t^{n}
$$

A.4.1. When $(R, \mathfrak{m}, k)$ is a local ring, each complex $M \in \mathrm{D}_{+}^{\mathrm{f}}(R)$ admits a resolution $F \stackrel{\cong}{\rightrightarrows} M$ with $F \in \mathrm{D}_{+}^{\mathrm{f}}(R)$, such that $\partial(F) \subseteq \mathfrak{m} F$ holds and each $F_{n}$ is free of finite rank; this forces $\inf F=\inf \mathrm{H}(M)$. Since $k \otimes_{R} F$ is a complex of $k$-vector spaces with zero differential, there are isomorphisms

$$
k \otimes_{R}^{\mathrm{L}} M \simeq k \otimes_{R} F \simeq \mathrm{H}\left(k \otimes_{R} F\right) \quad \text { in } \mathrm{D}(R),
$$

which imply equalities $\operatorname{rank}_{k} \operatorname{Tor}_{n}^{R}(k, M)=\operatorname{rank}_{R} F_{n}$ for all $n \in \mathbb{Z}$.

In A.4.2 and Lemma A.4.3 below, the ring $R$ is not assumed local.

A.4.2. For $M$ in $D_{+}^{f}(R)$ and $\mathfrak{p}$ in $\operatorname{Spec} R$ the conditions $\mathfrak{p} \in \operatorname{Supp} M$ and $P_{M_{\mathfrak{p}}}^{R_{\mathfrak{p}}}(t) \neq 0$ are equivalent; when they hold, one has $\operatorname{ord}\left(P_{M_{\mathfrak{p}}}^{R_{\mathfrak{p}}}(t)\right)=\inf \mathrm{H}\left(M_{\mathfrak{p}}\right)$.

Indeed, both assertions are immediate consequences of A.4.1.

Lemma A.4.3. Let $M$ and $N$ be complexes in $\mathrm{D}^{\mathrm{f}}(R)$ and $\mathfrak{p}$ be a prime ideal of $R$. If $\left(M \otimes_{R}^{\mathrm{L}} N\right)_{\mathfrak{p}}$ is in $\mathrm{D}_{+}\left(R_{\mathfrak{p}}\right)$, then so are $M_{\mathfrak{p}}$ and $N_{\mathfrak{p}}$, and there are equalities

$$
\begin{gathered}
P_{\left(M \otimes_{R}^{\llcorner} N\right)_{\mathfrak{p}}}^{R_{\mathfrak{p}}}(t)=P_{M_{\mathfrak{p}}}^{R_{\mathfrak{p}}}(t) \cdot P_{N_{\mathfrak{p}}}^{R_{\mathfrak{p}}}(t), \\
\inf \mathrm{H}\left(\left(M \otimes_{R}^{\mathrm{L}} N\right)_{\mathfrak{p}}\right)=\inf \mathrm{H}\left(M_{\mathfrak{p}}\right)+\inf \mathrm{H}\left(N_{\mathfrak{p}}\right) .
\end{gathered}
$$

Proof. In $\mathrm{D}\left(R_{\mathfrak{p}}\right)$ one has $\left(M \otimes_{R}^{\mathrm{L}} N\right)_{\mathfrak{p}} \simeq M_{\mathfrak{p}} \otimes_{R_{\mathfrak{p}}}^{\mathrm{L}} N_{\mathfrak{p}}$, so it suffices to treat the case when $(R, \mathfrak{p}, k)$ is local. Note the following isomorphisms of graded vector spaces:

$$
\begin{aligned}
\mathrm{H}\left(k \otimes_{R}^{\mathrm{L}}\left(M \otimes_{R}^{\mathrm{L}} N\right)\right) & \cong \mathrm{H}\left(\left(k \otimes_{R}^{\mathrm{L}} M\right) \otimes_{k}^{\mathrm{L}}\left(k \otimes_{R}^{\mathrm{L}} N\right)\right) \\
& \cong \mathrm{H}\left(k \otimes_{R}^{\mathrm{L}} M\right) \otimes_{k} \mathrm{H}\left(k \otimes_{R}^{\mathrm{L}} N\right) .
\end{aligned}
$$

The hypotheses and A.1 yield $\mathrm{H}_{n}\left(k \otimes_{R}^{\mathrm{L}}\left(M \otimes_{R}^{\mathrm{L}} N\right)\right)=0$ for $n \ll 0$, so the isomorphism implies that $k \otimes_{R}^{\mathrm{L}} M$ and $k \otimes_{R}^{\mathrm{L}} N$ are in $\mathrm{D}_{+}(R)$, and thus $M$ and $N$ are in $\mathrm{D}_{+}^{\mathrm{f}}(R)$ by A.3. When they are, for each $n \in \mathbb{Z}$ one has an isomorphism of $k$-vector spaces

$$
\begin{aligned}
\left(\mathrm{H}\left(k \otimes_{R}^{\mathrm{L}} M\right) \otimes_{k} \mathrm{H}\left(k \otimes_{R}^{\mathrm{L}} N\right)\right)_{n} & \cong \bigoplus_{i+j=n} \mathrm{H}_{i}\left(k \otimes_{R}^{\mathrm{L}} M\right) \otimes_{k} \mathrm{H}_{j}\left(k \otimes_{R}^{\mathrm{L}} N\right) \\
& \cong \bigoplus_{i+j=n} \operatorname{Tor}_{i}^{R}(k, M) \otimes_{k} \operatorname{Tor}_{j}^{R}(k, N) .
\end{aligned}
$$

By equating the generating series for the ranks over $k$, we get the desired equality of Poincaré series; comparing orders and using A.4.2 gives the second equality. 
A.5 (Bass series). For a local ring $(R, \mathfrak{m}, k)$ and for $N$ in $\mathrm{D}_{-}^{f}(R)$, in view of A.1 the following formula defines a formal Laurent series, called the Bass series of $N$ :

$$
I_{R}^{N}(t)=\sum_{n \in \mathbb{Z}} \operatorname{rank}_{k} \operatorname{Ext}_{R}^{n}(k, N) t^{n}
$$

A.5.1. For a local ring $R$ and $N$ in $\mathrm{D}_{-}^{\mathrm{f}}(R)$ one has $\operatorname{ord}\left(I_{R}^{N}(t)\right)=\operatorname{depth}_{R} N$; this follows from the definition of depth (see Section 1). Furthermore, $I_{R}^{N}(t)$ is a Laurent polynomial if and only if $N$ has finite injective dimension; see, for example, [Avramov and Foxby 1991, 5.5].

In the remaining statements the ring $R$ is not necessarily local.

A.5.2. For $N$ in $D_{-}^{f}(R)$ and $\mathfrak{p}$ in $\operatorname{Spec} R$, the conditions $\mathfrak{p} \in \operatorname{Supp} N$ and $I_{R_{\mathfrak{p}}}^{N_{\mathfrak{p}}}(t) \neq 0$ are equivalent; when they hold, one has $\operatorname{ord}\left(I_{R_{\mathfrak{p}}}^{N_{\mathfrak{p}}}(t)\right)=\operatorname{depth}_{R_{\mathfrak{p}}} N_{\mathfrak{p}}$.

Indeed, in view of A.5.1, the assertions follow from the fact that depth $R_{\mathfrak{p}} N_{\mathfrak{p}}<\infty$ is equivalent to $\mathrm{H}\left(N_{\mathfrak{p}}\right) \neq 0$; see, for instance, [Foxby and Iyengar 2003, 2.5].

Lemma A.5.3. Let $M$ and $N$ be complexes in $\mathrm{D}^{\mathrm{f}}(R)$ and $\mathfrak{p}$ a prime ideal of $R$. If $\mathrm{RHom}_{R}(M, N)$ is in $\mathrm{D}_{-}(R)$ then $M_{\mathfrak{p}}$ is in $\mathrm{D}_{+}^{\mathrm{f}}\left(R_{\mathfrak{p}}\right)$.

If, in addition, $\mathfrak{p}$ is the unique maximal ideal of $R$, or $\mathfrak{p}$ is maximal and $N$ is in $D_{-}^{f}(R)$, or $M$ is in $D_{+}^{f}(R)$ and $N$ is in $D_{-}^{f}(R)$, then there are equalities

$$
\begin{aligned}
& I_{R_{\mathfrak{p}}}^{\mathrm{RHom}} \operatorname{Rom}_{R}(M, N)_{\mathfrak{p}}(t)=P_{M_{\mathfrak{p}}}^{R_{\mathfrak{p}}}(t) \cdot I_{R_{\mathfrak{p}}}^{N_{\mathfrak{p}}}(t), \\
& \operatorname{depth}_{R_{\mathfrak{p}}}\left(\operatorname{RHom}_{R}(M, N)_{\mathfrak{p}}\right)=\inf \left(\mathrm{H}\left(M_{\mathfrak{p}}\right)\right)+\operatorname{depth}_{R_{\mathfrak{p}}}\left(N_{\mathfrak{p}}\right) \text {. }
\end{aligned}
$$

Proof. Assume first that $\mathfrak{p}$ is maximal and set $k=R / \mathfrak{p}$. One gets isomorphisms

$$
\begin{aligned}
\mathrm{H}\left(\operatorname{RHom}_{R}\left(k, \operatorname{RHom}_{R}(M, N)\right)\right) & \cong \mathrm{H}\left(\operatorname{RHom}_{R}\left(k \otimes_{R}^{\mathrm{L}} M, N\right)\right) \\
& \cong \mathrm{H}\left(\operatorname{RHom}_{k}\left(k \otimes_{R}^{\mathrm{L}} M, \operatorname{RHom}_{R}(k, N)\right)\right) \\
& \cong \operatorname{Hom}_{k}\left(\mathrm{H}\left(k \otimes_{R}^{\mathrm{L}} M\right), \operatorname{H}_{\left.\left(\operatorname{RHom}_{R}(k, N)\right)\right)}\right.
\end{aligned}
$$

of graded $k$-vector spaces by using standard maps. In view of A.1, for $n \gg 0$ one has $\mathrm{H}_{n}\left(\operatorname{RHom}_{R}\left(k, \operatorname{RHom}_{R}(M, N)\right)\right)=0$, so the isomorphisms yield $k \otimes_{R}^{\mathrm{L}} M \in \mathrm{D}_{+}(R)$ and $\operatorname{RHom}_{R}(k, N) \in \mathrm{D}_{-}(R)$. When $R$ is local, one gets $M \in \mathrm{D}_{+}^{\mathrm{f}}(R)$ and $N \in \mathrm{D}_{-}^{\mathrm{f}}(R)$ from A.3. For general $R$, this implies $M_{\mathfrak{p}} \in \mathrm{D}_{+}^{f}\left(R_{\mathfrak{p}}\right)$ in view of the isomorphism $k \otimes_{R}^{\mathrm{L}} M \simeq k \otimes_{R_{\mathfrak{p}}}^{\mathrm{L}} M_{\mathfrak{p}}$ from A.2. If $N$ is in $\mathrm{D}_{-}^{\mathrm{f}}(R)$, then by referring once more to loc. cit. we can rewrite the isomorphisms above in each degree $n$ in the form

$$
\begin{aligned}
\operatorname{Ext}_{R_{\mathfrak{p}}}^{n}\left(k, \operatorname{RHom}_{R}(M, N)_{\mathfrak{p}}\right) & \cong \operatorname{Hom}_{k}\left(\operatorname{Tor}^{R_{\mathfrak{p}}}\left(k, M_{\mathfrak{p}}\right), \operatorname{Ext}_{R_{\mathfrak{p}}}\left(k, N_{\mathfrak{p}}\right)\right)_{-n} \\
& \cong \bigoplus_{i-j=n} \operatorname{Hom}_{k}\left(\operatorname{Tor}_{i}^{R_{\mathfrak{p}}}\left(k, M_{\mathfrak{p}}\right), \operatorname{Ext}_{R_{\mathfrak{p}}}^{-j}\left(k, N_{\mathfrak{p}}\right)\right) .
\end{aligned}
$$


For the generating series for the ranks over $k$ these isomorphisms give

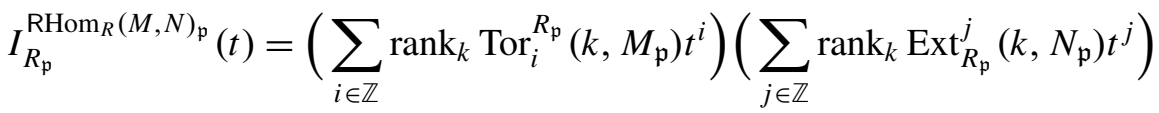

$$
\begin{aligned}
& =P_{M_{\mathfrak{p}}}^{R_{\mathfrak{p}}}(t) \cdot I_{R_{\mathfrak{p}}}^{N_{\mathfrak{p}}}(t) .
\end{aligned}
$$

Equating orders of formal Laurent and using A.5.2 one gets the second equality.

Let now $\mathfrak{p}$ be an arbitrary prime ideal and $\mathfrak{m}$ a maximal ideal containing $\mathfrak{p}$. The preceding discussion shows that $M_{\mathfrak{m}}$ is in $\mathrm{D}_{+}^{\mathrm{f}}\left(R_{\mathfrak{m}}\right)$, hence $M_{\mathfrak{p}}$ is in $\mathrm{D}_{+}^{\mathrm{f}}\left(R_{\mathfrak{p}}\right)$. When $M$ is in $\mathrm{D}_{+}^{\mathrm{f}}(R)$ and $N$ is in $\mathrm{D}_{-}^{\mathrm{f}}(R)$ one has $\operatorname{RHom}_{R}(M, N)_{\mathfrak{p}} \cong \operatorname{RHom}_{R_{\mathfrak{p}}}\left(M_{\mathfrak{p}}, N_{\mathfrak{p}}\right)$, so the desired equalities follow from those that have already been established.

A.6. The support of a complex $M$ in $\mathrm{D}_{\mathrm{b}}^{\mathrm{f}}(R)$ is the set

$$
\operatorname{Supp}_{R} M=\left\{\mathfrak{p} \in \operatorname{Spec} R \mid \mathrm{H}(M)_{\mathfrak{p}} \neq 0\right\} .
$$

One has $\operatorname{Supp}_{R} M=\varnothing$ if and only if $\mathrm{H}(M)=0$, if and only if $M \simeq 0$ in $\mathrm{D}(R)$.

For all complexes $M, N$ in $\mathrm{D}_{\mathrm{b}}^{\mathrm{f}}(R)$ there are equalities

$$
\operatorname{Supp}_{R}\left(M \otimes_{R}^{\mathrm{L}} N\right)=\operatorname{Supp}_{R} M \cap \operatorname{Supp}_{R} N=\operatorname{Supp}_{R} \operatorname{RHom}_{R}(M, N) .
$$

This follows directly from A.4.2, A.5.2, and Lemmas A.4.3 and A.5.3.

\section{Acknowledgments}

We are grateful to Lars Winther Christensen, Amnon Neeman, and Sean SatherWagstaff for their comments and suggestions on earlier versions of this article.

\section{References}

[Auslander and Bridger 1969] M. Auslander and M. Bridger, Stable module theory, Memoirs of the American Mathematical Society 94, American Mathematical Society, Providence, R.I., 1969. MR 42 \#4580 Zbl 0204.36402

[Avramov and Foxby 1991] L. L. Avramov and H.-B. Foxby, "Homological dimensions of unbounded complexes”, J. Pure Appl. Algebra 71:2-3 (1991), 129-155. MR 93g:18017 Zbl 0737. 16002

[Avramov and Foxby 1997] L. L. Avramov and H.-B. Foxby, "Ring homomorphisms and finite Gorenstein dimension”, Proc. London Math. Society (3) 75:2 (1997), 241-270. MR 98d:13014 Zbl 0901.13011

[Avramov and Iyengar 2008] L. L. Avramov and S. B. Iyengar, "Gorenstein algebras and Hochschild cohomology”, Michigan Math. J. 57 (2008), 17-35. MR 2009k:13047 Zbl 05604517

[Avramov et al. 2010a] L. L. Avramov, S. B. Iyengar, J. Lipman, and S. Nayak, "Reduction of derived Hochschild functors over commutative algebras and schemes", Adv. Math. 30 (2010), 735772. arXiv 0904.4004

[Avramov et al. 2010b] L. L. Avramov, S. B. Iyengar, and J. Lipman, "Reflexivity and rigidity for complexes, II: Schemes", preprint, 2010. arXiv 1001.3450 
[Bass and Murthy 1967] H. Bass and M. P. Murthy, "Grothendieck groups and Picard groups of abelian group rings”, Ann. of Math. (2) 86 (1967), 16-73. MR 36 \#2671 Zbl 0157.08202

[van den Bergh 1997] M. van den Bergh, "Existence theorems for dualizing complexes over noncommutative graded and filtered rings", J. Algebra 195:2 (1997), 662-679. MR 99b:16010 Zbl 0894.16020

[Chouinard 1976] L. G. Chouinard, II, "On finite weak and injective dimension", Proc. Amer. Math. Soc. 60 (1976), 57-60. MR 54 \#5217 Zbl 0343.13005

[Christensen 2000] L. W. Christensen, Gorenstein dimensions, Lecture Notes in Mathematics 1747, Springer, Berlin, 2000. MR 2002e:13032 Zbl 0965.13010

[Christensen 2001] L. W. Christensen, "Semi-dualizing complexes and their Auslander categories", Trans. Amer. Math. Soc. 353:5 (2001), 1839-1883. MR 2002a:13017 Zbl 0969.13006

[Christensen et al. 2002] L. W. Christensen, H.-B. Foxby, and A. Frankild, "Restricted homological dimensions and Cohen-Macaulayness", J. Algebra 251:1 (2002), 479-502. MR 2003e:13022 Zbl 1073.13501

[Conrad 2000] B. Conrad, Grothendieck duality and base change, Lecture Notes in Mathematics 1750, Springer, Berlin, 2000. MR 2002d:14025 Zbl 0992.14001

[Foxby 1972] H.-B. Foxby, "Gorenstein modules and related modules", Math. Scand. 31 (1972), 267-284. MR 48 \#6094 Zbl 0272.13009

[Foxby 1977] H.-B. Foxby, "Isomorphisms between complexes with applications to the homological theory of modules", Math. Scand. 40:1 (1977), 5-19. MR 56 \#5584 Zbl 0356.13004

[Foxby 1979] H.-B. Foxby, "Bounded complexes of flat modules", J. Pure Appl. Algebra 15:2 (1979), 149-172. MR 83c:13008 Zbl 0411.13006

[Foxby and Iyengar 2003] H.-B. Foxby and S. Iyengar, "Depth and amplitude for unbounded complexes", pp. 119-137 in Commutative algebra (Grenoble/Lyon, 2001), edited by L. L. Avramov et al., Contemp. Math. 331, Amer. Math. Soc., Providence, RI, 2003. MR 2004k:13028 Zbl 1096. 13516

[Frankild and Sather-Wagstaff 2007] A. Frankild and S. Sather-Wagstaff, "Reflexivity and ring homomorphisms of finite flat dimension", Comm. Algebra 35:2 (2007), 461-500. MR 2007m:13022 Zbl 1118.13015

[Frankild et al. 2009] A. J. Frankild, S. Sather-Wagstaff, and A. Taylor, "Relations between semidualizing complexes”, J. Commut. Algebra 1:3 (2009), 393-436. MR 2524860

[Golod 1984] E. S. Golod, "G-dimension and generalized perfect ideals", pp. 62-66 in Algebraic geometry and its applications, Trudy Mat. Inst. Steklov. 165, 1984. In Russian; translated in Proc. Steklov Inst. Math. 165 (1985), 62-66. MR 85m:13011 Zbl 0577.13008

[Goto 1982] S. Goto, "Vanishing of $\operatorname{Ext}_{A}^{i}(M, A)$ ", J. Math. Kyoto Univ. 22:3 (1982), 481-484. MR 84c:13019 Zbl 0527.13012

[Grothendieck 1967] A. Grothendieck, "Éléments de géométrie algébrique, IV: Étude locale des schémas et des morphismes de schémas IV", Inst. Hautes Études Sci. Publ. Math. 32 (1967), 361. MR 39 \#220 Zbl 0153.22301

[Hartshorne 1966] R. Hartshorne, Residues and duality, Lecture notes of a seminar on the work of A. Grothendieck, given at Harvard 1963/64. With an appendix by P. Deligne. Lecture Notes in Mathematics, No. 20, Springer, Berlin, 1966. MR 36 \#5145 Zbl 0212.26101

[Ischebeck 1969] F. Ischebeck, "Eine Dualität zwischen den Funktoren Ext und Tor", J. Algebra 11 (1969), 510-531. MR 38 \#5894 
[Kawasaki 2002] T. Kawasaki, “On arithmetic Macaulayfication of Noetherian rings", Trans. Amer. Math. Soc. 354:1 (2002), 123-149. MR 2002i:13001 Zbl 1087.13502

[Lipman 1984] J. Lipman, Dualizing sheaves, differentials and residues on algebraic varieties, Astérisque 117, 1984. MR 86g:14008 Zbl 0562.14003

[Matsumura 1986] H. Matsumura, Commutative ring theory, Cambridge Studies in Advanced Math. 8, Cambridge University Press, Cambridge, 1986. MR 88h:13001 Zbl 0603.13001

[Neeman 2008] A. Neeman, "Derived categories and Grothendieck duality", 2008, Available at www.crm.es/Publications/08/Pr791.pdf. To appear in Triangulated categories (Leeds, 2006), London Math. Soc. Lecture Note Ser. Cambridge University Press.

[Quillen 1970] D. Quillen, "On the (co-) homology of commutative rings", pp. 65-87 in Applications of categorical algebra (New York, 1968), edited by A. Heller, Proc. Sympos. Pure Math. 17, Amer. Math. Soc., Providence, R.I., 1970. MR 41 \#1722 Zbl 0234.18010

[Yekutieli 1999] A. Yekutieli, "Dualizing complexes, Morita equivalence and the derived Picard group of a ring”, J. London Math. Soc. (2) 60:3 (1999), 723-746. MR 2001i:16008 Zbl 0954.16006

[Yekutieli and Zhang 2008] A. Yekutieli and J. J. Zhang, "Rigid complexes via DG algebras", Trans. Amer. Math. Soc. 360:6 (2008), 3211-3248. MR 2008m:16016 Zbl 1165.18009

[Yekutieli and Zhang 2009] A. Yekutieli and J. J. Zhang, "Rigid dualizing complexes over commutative rings", Algebr. Represent. Theory 12:1 (2009), 19-52. MR 2471449 Zbl 05619084

Communicated by Craig Huneke

Received 2009-04-15 Revised 2009-07-15 Accepted 2009-08-18

avramov@math.unl.edu

iyengar@math.unl.edu

jlipman@purdue.edu
Department of Mathematics, University of Nebraska, Lincoln, NE 68588, United States

Department of Mathematics, University of Nebraska, Lincoln, NE 68588, United States www. math.unl.edu/ siyengar2

Department of Mathematics, Purdue University, W. Lafayette, IN 47907, United States 